\title{
Sustained inflammation after pericyte depletion induces irreversible blood-retina barrier breakdown
}

\author{
Shuntaro Ogura, ${ }^{1,2}$ Kaori Kurata, ${ }^{1}$ Yuki Hattori, ${ }^{3}$ Hiroshi Takase, ${ }^{4}$ Toshina Ishiguro-Oonuma, ${ }^{5}$ \\ Yoonha Hwang, ${ }^{6}$ Soyeon Ahn, ${ }^{6}$ Inwon Park, ${ }^{7}$ Wataru Ikeda, ${ }^{8}$ Sentaro Kusuhara, ${ }^{9}$ Yoko Fukushima, ${ }^{10}$ \\ Hiromi Nara, ${ }^{11}$ Hideto Sakai, ${ }^{11}$ Takashi Fujiwara, ${ }^{12}$ Jun Matsushita, ${ }^{13}$ Masatsugu Ema, ${ }^{13}$ \\ Masanori Hirashima, ${ }^{14}$ Takashi Minami, ${ }^{15}$ Masabumi Shibuya, ${ }^{16}$ Nobuyuki Takakura, ${ }^{17}$ \\ Pilhan Kim, ${ }^{6}$ Takaki Miyata, ${ }^{3}$ Yuichiro Ogura, ${ }^{2}$ and Akiyoshi Uemura, ${ }^{1,2}$ \\ 'Department of Retinal Vascular Biology, ${ }^{2}$ Department of Ophthalmology and Visual Sciences, Nagoya City University \\ Graduate School of Medical Sciences, Nagoya, Japan. ${ }^{3}$ Department of Anatomy and Cell Biology, Nagoya University \\ Graduate School of Medicine, Nagoya, Japan. ${ }^{4}$ Core Laboratory, Nagoya City University Graduate School of Medical \\ Sciences, Nagoya, Japan. 'Laboratory of Veterinary Physiology, Cooperative Department of Veterinary Medicine, Faculty \\ of Agriculture, Iwate University, Morioka, Japan. ${ }^{6}$ Graduate School of Nanoscience and Technology, and ${ }^{7}$ Graduate School \\ of Medical Science and Engineering, Korea Advanced Institute of Science and Technology, Daejeon, Republic of Korea. \\ ${ }^{8}$ KAN Research Institute, Kobe, Japan. ${ }^{9}$ Division of Ophthalmology, Department of Surgery, Kobe University Graduate \\ School of Medicine, Kobe, Japan. ${ }^{10}$ Department of Ophthalmology, Osaka University Graduate School of Medicine, Osaka, \\ Japan. "'Drug Discovery Research, Astellas Pharma Inc., Tsukuba, Japan. ${ }^{12}$ Faculty of Nursing, Hiroshima Bunka Gakuen \\ University, Kure, Japan. ${ }^{13}$ Department of Stem Cells and Human Disease Models, Research Center for Animal Life Science, \\ Shiga University of Medical Science, Otsu, Japan. ${ }^{14}$ Division of Vascular Biology, Department of Physiology and Cell \\ Biology, Kobe University Graduate School of Medicine, Kobe, Japan. ${ }^{15}$ Division of Phenotype Disease Analysis, Institute of \\ Resource Development and Analysis, Kumamoto University, Kumamoto, Japan. ${ }^{16}$ Institute of Physiology and Medicine, \\ Jobu University, Takasaki, Japan. ${ }^{17}$ Department of Signal Transduction, Research Institute for Microbial Diseases, Osaka \\ University, Osaka, Japan.
}

In the central nervous system, endothelial cells (ECs) and pericytes (PCs) of blood vessel walls cooperatively form a physical and chemical barrier to maintain neural homeostasis. However, in diabetic retinopathy (DR), the loss of PCs from vessel walls is assumed to cause breakdown of the blood-retina barrier (BRB) and subsequent vision-threatening vascular dysfunctions. Nonetheless, the lack of adequate DR animal models has precluded disease understanding and drug discovery. Here, by using an anti-PDGFR $\beta$ antibody, we show that transient inhibition of the PC recruitment to developing retinal vessels sustained EC-PC dissociations and BRB breakdown in adult mouse retinas, reproducing characteristic features of DR such as hyperpermeability, hypoperfusion, and neoangiogenesis. Notably, PC depletion directly induced inflammatory responses in ECs and perivascular infiltration of macrophages, whereby macrophage-derived VEGF and placental growth factor (PIGF) activated VECFR1 in macrophages and VEGFR2 in ECs. Moreover, angiopoietin-2 (Angpt2) upregulation and Tie1 downregulation activated FOX01 in PC-free ECs locally at the leaky aneurysms. This cycle of vessel damage was shut down by simultaneously blocking VEGF, PICF, and Angpt2, thus restoring the BRB integrity. Together, our model provides new opportunities for identifying the sequential events triggered by PC deficiency, not only in DR, but also in various neurological disorders.

Conflict of interest: W. Ikeda is an employee of the KAN Research Institute. H. Nara and H. Sakai are employees of Astellas Pharma Inc.

Submitted: September 26, 2016 Accepted: December 15, 2016 Published: February 9, 2017

Reference information: JCI Insight. 2017;2(3):e90905. https:// doi.org/10.1172/jici.insight.90905.

Authorship note: S. Ogura and K. Kurata contributed equally to this work.

\section{Introduction}

In the cardiovascular system, pericytes (PCs) maintain the integrity of blood vessel walls, not only by providing mechanical support, but also by reciprocally communicating with endothelial cells (ECs) via secretory signals and direct cell-to-cell contacts (1). Thus, PCs play pivotal roles in the control of vascular development and homeostasis. While PCs are functionally heterogeneous depending on the tissue, ECs and PCs in the central nervous system (CNS) cooperatively form a physical and chemical barrier that tightly controls the passage of 
fluids, molecules, and ions, thereby maintaining the proper environment for neurons and glial cells, and protecting them from toxins and pathogens (2). In certain CNS disorders such as Alzheimer's disease, PC deficiency is implicated in barrier disruptions during disease progression (3). Especially in diabetic retinopathy (DR), PC loss from capillary walls is assumed to be an initial pathological change responsible for the breakdown of the blood-retina barrier (BRB) and subsequent vascular hyperpermeability (4). In more advanced forms of DR, vessel obstruction generates retinal hypoperfusion and hypoxia, leading to aberrant formation of new blood vessels that directly cause blindness from vitreous hemorrhage and tractional retinal detachment (5). However, because hyperglycemic animal models fail to fully mimic the pathophysiology of human DR, molecular and cellular mechanisms underlying the barrier dysfunctions in PC-free retinal vessels remain elusive.

To evaluate the direct effects of PC depletion on retinal vessel integrity, we previously used a postnatal angiogenesis model in mouse retinas, in which new blood vessels radially grow in the superficial layer until P8-P10, then sprout downward at approximately P7 to form deep and intermediate vascular layers (6). In this process, ECs at the tips of sprouting vessels express PDGF-B to promote proliferation and migration of PDGFR $\beta$-expressing PCs along the nascent vessels (1). Consequently, most of the ECs associate with PCs over the developing retinal vasculature, with PCs acquiring heterogeneous properties depending on the vessel type (7). For example, PCs uniformly express PDGFR $\beta$, desmin, and NG2 proteoglycan, while $\alpha$-smooth muscle actin ( $\alpha$ SMA) is expressed strongly in arteries and weakly in veins, but not in capillaries (7). Nevertheless, in all types of retinal vessels, PC recruitment was completely inhibited by daily administration of an antagonistic anti-PDGFR $\beta$ mAb to neonatal mice (7). Importantly, PC deficiency was sufficient to reproduce the retinal vascular abnormalities characteristic of DR. However, retinal collapse resulting from progressive edema and hemorrhage prevented the assessment of sequential events triggered by PC depletion.

Despite limitations of the experimental animal models, clinical efficacy of anti-VEGF drugs and corticosteroids for diabetic macular edema has empirically shown the involvement of the VEGF signal and inflammation in the BRB breakdown in DR (8). In both physiological and pathological settings, VEGF is upregulated under hypoxia, and activates VEGFR2 on ECs to promote cell proliferation and migration (9). Furthermore, the VEGF/VEGFR2 signaling pathway facilitates the degradation of vascular endothelial (VE) cadherin and loosens the intercellular junctions between neighboring ECs (10). Thus, the VEGF/ VEGFR2 signal plays a predominant role in angiogenesis and vascular leakage. In contrast, VEGFR1 activation is assumed to be negligible in angiogenic ECs. Instead, activation of VEGFR1 in macrophages (MPs) by VEGF, or by placental growth factor (P1GF), contributes to the exacerbation of certain pathophysiological conditions such as inflammation (11). Although the protein expression levels of VEGF and PlGF, as well as inflammatory cytokines such as TNF- $\alpha$, IL-6, and CCL2, are highly upregulated in eyes with DR (12-14), it remains unclear how these signals are related in the PC-free retinas.

Regarding retinal vessel integrity, attention has been focused on signals mediated by angiopoietin (Angpt) ligands and Tie2 receptor tyrosine kinase (15). In ECs, binding of PC-derived Angpt1 to Tie2 activates the PI3K/Akt pathway, and inactivates the forkhead transcription factor, FOXO1, thereby promoting cell survival, strengthening intercellular junctions, and suppressing inflammation (16). Angpt2 counteracts these vessel-stabilizing effects as a natural antagonist for Tie2, and renders ECs more sensitive to stimuli from the microenvironment, including VEGF and TNF- $\alpha$ (16). Notably, hypoxia and VEGF upregulate transcriptional levels of the Angpt2 gene in ECs $(17,18)$, whereas Tie2 inactivation by Angpt2 leads to the nuclear translocation of FOXO1, which then further upregulates Angpt2 (19). Thus, Angpt2 acts as a facilitator of angiogenesis, vascular permeability, and inflammation in a variety of pathological settings. However, Angpt 2 can also act as a Tie2 agonist depending on its environment, especially at high concentrations (20) and in stressed ECs (21). Of importance, VEGF and TNF- $\alpha$ promote proteolytic cleavage of the orphan receptor Tie1, thereby switching Anpgt2 from a Tie2 agonist to an antagonist (22-24). In eyes with DR, the expression levels of Angpt2 proteins are significantly upregulated (25), but its mode of action is still obscure.

In the present study, we show that partial inhibition of PC recruitment to developing retinal vessels circumvented the retinal collapse, but the EC-PC associations failed to be spontaneously normalized even in adult retinas. PC depletion directly induced inflammatory responses in ECs and perivascular infiltration of VEGFR $1^{+}$MPs, and the ECs and MPs underwent a cycle of vessel damage via VEGF, P1GF, and Angpt2. Thus, our experimental model facilitates a new means to understand how PC deficiency in retinas initiates the sustained inflammation and irreversible BRB breakdown, and provides a potentially new drug discovery system, not only for DR, but also for neurological disorders. 


\section{Results}

Optional inhibition of PC recruitment to retinal vessels by transient PDGFR $\beta$ blockade. We previously reported that i.p. injections of $100 \mu \mathrm{g}$ of an anti-PDGFR $\beta$ mAb (clone APB5) daily from P0 completely inhibited $\mathrm{PC}$ recruitment to developing retinal vessels, resulting in retinal collapse at P10 (7). To avoid this collapse, we optimized the dose, frequency, and timing of i.p. APB5 injections, and found that even a single injection of $50 \mu \mathrm{g}$ of APB5 at P1 resulted in retinal collapse at P10 (data not shown). Thereafter, we decreased the amount of APB5 used for a single injection at P1. As expected, these treatments reduced PC coverage in a dose-dependent manner, with almost complete depletion of capillary PCs at P5 using $40 \mu \mathrm{g}$ of APB5 (Figure 1, A and B). In contrast, in retinas treated with $20 \mu \mathrm{g}$ of APB5, PCs discontinuously covered capillaries with upregulated $\alpha$ SMA (Figure 1, A and B). In accordance with the extent of PC depletions, all types of retinal vessels were enlarged, with decreased vascular extensions and increased vessel densities (Figure 1, A and B, and Supplemental Figure 1A; supplemental material available online with this article; https/doi.org/10.1172/jci.insight.90905DS1).

In growing retinal vessels, specialized EC properties are crucial for efficient vessel elongation; the tip ECs project filopodia while the following stalk ECs proliferate (26). However, in the absence of PCs, even the tip ECs proliferated in the blunt vascular sprouts, whereas their filopodia projections were significantly decreased (Figure 1C). On the other hand, a tip EC marker, EC-specific molecule 1, was ectopically expressed in stalk ECs (Figure 1D), suggesting disturbed tip and stalk properties in PC-free ECs. Notably, in the APB5-treated retinas, the ECs lost their spindle shapes and were disarranged in all types of vessels (Supplemental Figure 1B). Furthermore, PC-free ECs in preformed vessels maintained high mitotic activities but failed to sprout downward into the deep retinal layers, resulting in the formation of aneurysm-like structures at P7-P8 (Figure 1, E and F, and Supplemental Video 1), with increased EC apoptosis (Figure $1 G)$. Together, these findings suggested that in the absence of PCs, the ECs were continuously sensitive to their microenvironments, whereby blood vessels were subject to dysregulated remodeling.

In addition to these morphological defects, we investigated the barrier function of PC-free retinal vessels. After a single injection of $20 \mu \mathrm{g}$ of APB5, more than $80 \%$ of the retinal samples showed no obvious hemorrhage and edema at P10 (Supplemental Figure 1C). In contrast, injections of $40 \mu \mathrm{g}$ of APB5 resulted in progressive vascular leakage and hemorrhage at P8 (Figure 1, H and I). Importantly, the tracer perfusion experiments showed focal, but not diffuse, leakage from PC-free vessels, especially from the aneurysmlike structures (Figure $1 \mathrm{~J}$ ). Subsequently, $60 \%$ of the retinas collapsed at P10 (Supplemental Figure 1C), whereas the remaining samples showed edema and hemorrhage in localized retinal areas, with exudative depositions (Figure 1, K-M). Notably, the proportion of retinal collapse induced by $40 \mu \mathrm{g}$ of APB5 was unchanged at later stages (data not shown), indicating P10 as a turning point in our model.

To further determine the leakage routes, we labeled adherens and tight junctions of ECs with VEcadherin and claudin-5, respectively (10). In APB5-treated retinas, VE-cadherin staining showed interdigitated but continuous adherens junctions, whereas claudin-5 expression was barely detectable (Figure $1 \mathrm{~N}$ ). We next examined the expression of plasmalemma vesicle-associated protein (PLVAP), a marker for a key structural element of vesicular transport in ECs (27). In contrast to the absence of its expression in the control retinas, PLVAP was highly expressed in PC-free ECs (Figure 1O). These results indicated elevation of both paracellular and transcellular leakage in PC-free retinal vessels. This was further supported by electron microscopy that showed the uptake of i.v. perfused biotin tracers both at discontinuous intercellular junctions and in intracellular vesicles in PC-free ECs (Figure 1P), with local rupture of their luminal surfaces (Supplemental Figure 1D).

An adult mouse model reproducing characteristics of $D R$. Even in noncollapsed retinas treated with $30 \mu \mathrm{g}$ of APB5, the disorganized vascular networks failed to be spontaneously normalized, with increased frequency of artery-vein crossings in the superficial vascular networks, and underdevelopment of intermediate and deep vascular networks (Figure 2A and Supplemental Figure 2A). At 4 weeks, APB5-treated retinas showed sustained hemorrhage with scattered yellowish spots (Figure 2B), resembling hard exudates resulting from the deposition of extravasated lipoproteins in DR (5) (Figure 2C). In addition, angiography showed leakage and nonperfusion of systemically administered fluorescein dye in APB5-treated retinas (Figure 2D), which was characteristic of DR (Figure 2E). Optical coherence tomography further showed intra- and subretinal fluid accumulation (Figure 2F), similar to DR (Figure 2G), which was also shown by histological examination (Figure 2H). Accordingly, the thickness of APB5-treated retinas was significantly increased, but that of their outer nuclear layers containing photoreceptor cells was significantly 
A

A Control APB5 $20 \mu \mathrm{g} \quad$ APB5 $40 \mu \mathrm{g} \quad$ B
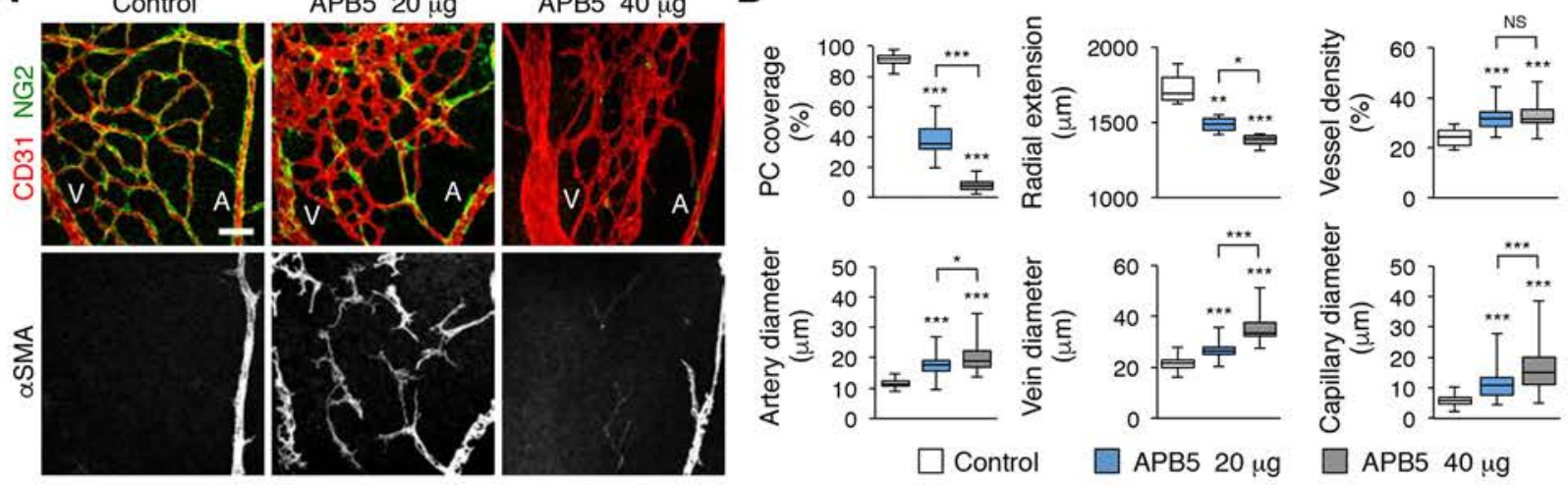

C
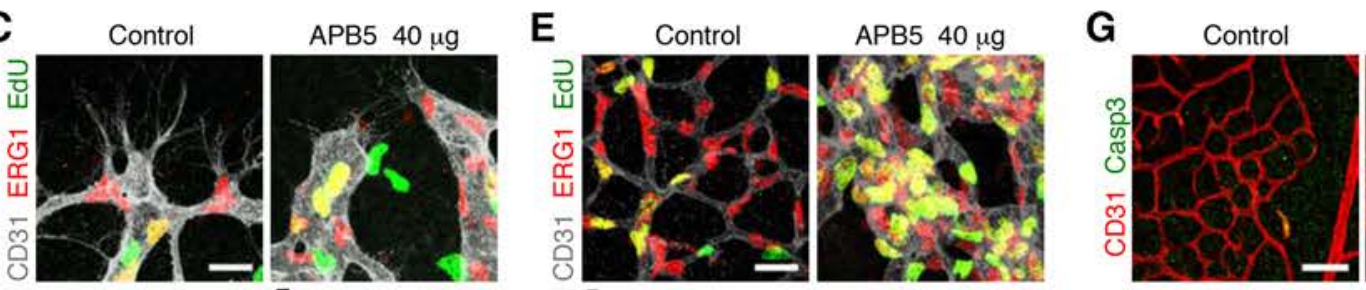

APB5 $40 \mu \mathrm{g}$
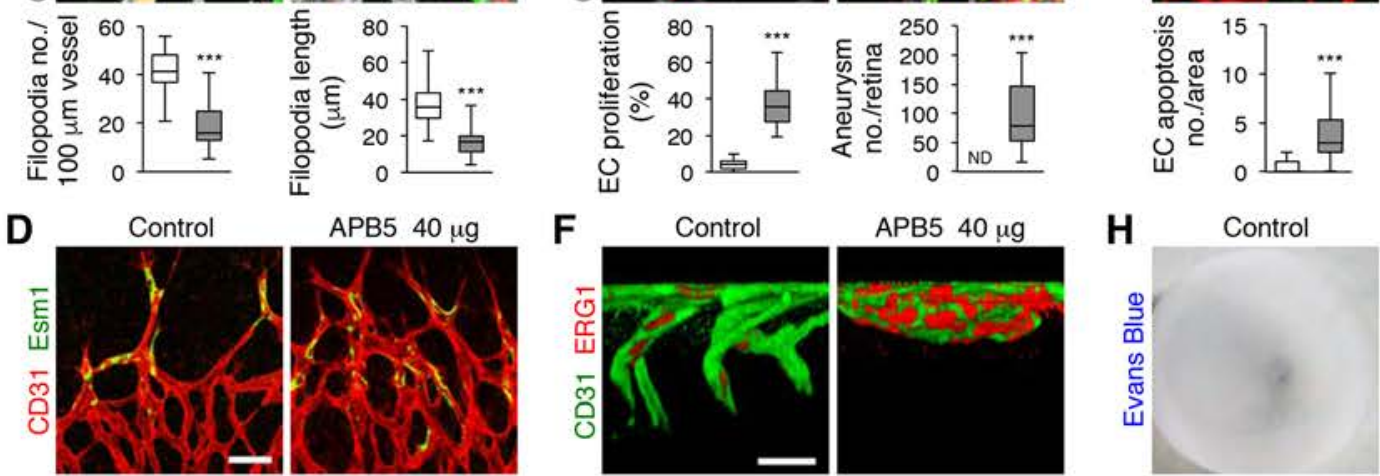

APB5 $40 \mu \mathrm{g}$
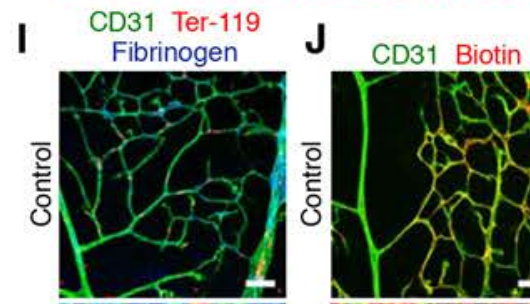

$\mathbf{K}$

L

M CD31 Fibrinogen
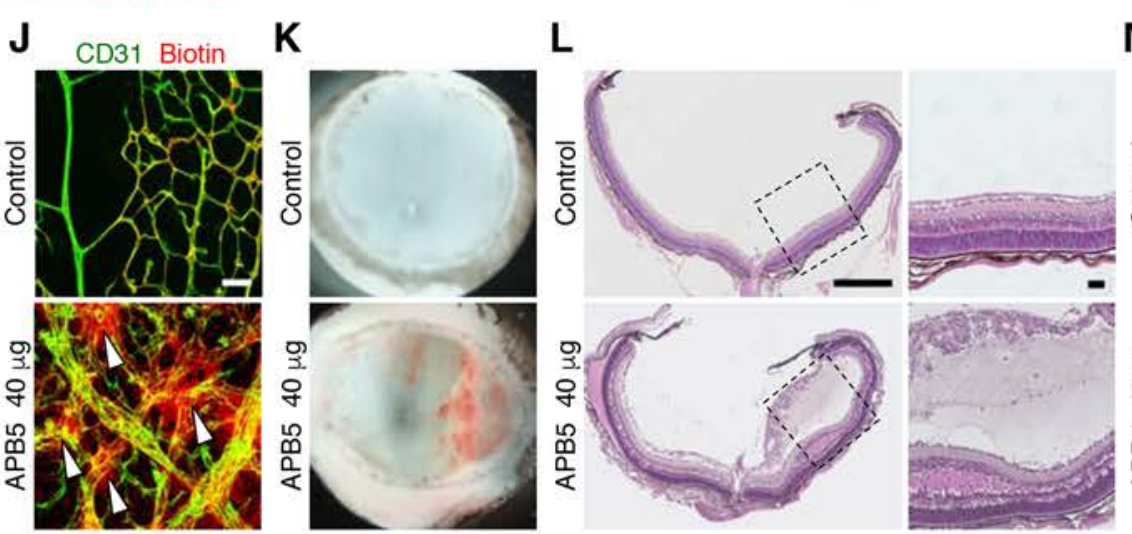

$\square$ Control

$\square$ APB5 $40 \mu \mathrm{g}$

\section{.}

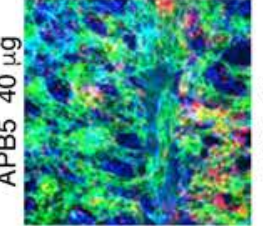

Claudin-5

N VE-cad ERG1
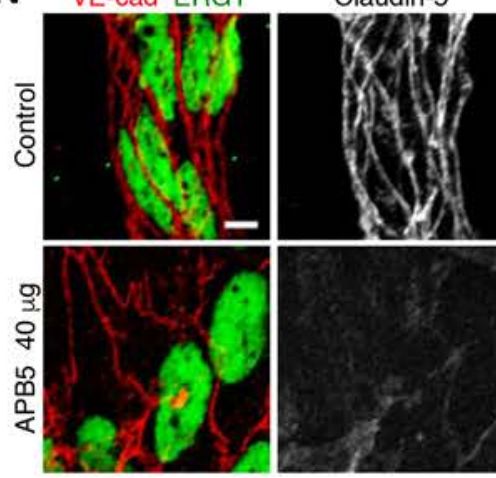

0

CD31

PLVAP

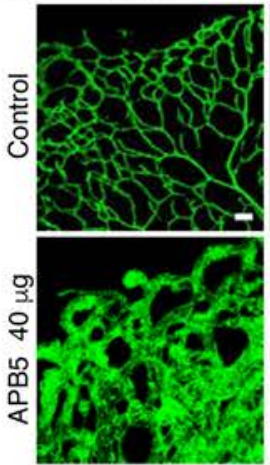

NG2

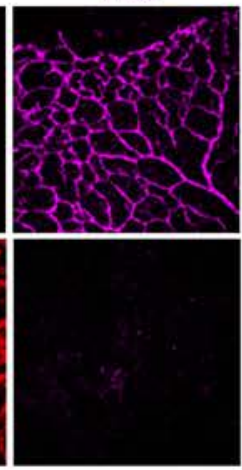

$\mathbf{P}$

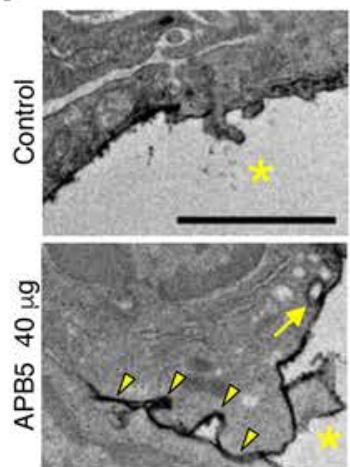


Figure 1. Inhibition of pericyte (PC) recruitment during retinal vascular development. (A) IHC for CD31, NC2, and $\alpha$-smooth muscle actin ( $\alpha$ SMA) at P5. A, artery; V, vein. (B) Quantification of PC coverage ( $n=24$ areas), radial vascular extension ( $n=6$ retinas), vessel density $(n=24$ areas), and diameters of arteries $(n=30)$, veins $(n=24)$, and capillaries $(n=120)$ in P5 retinas. (C) Labeling for CD31, Ets-related gene-1 (ERG1), and 5-ethynyl-2'-deoxyuridine (EdU) at the sprouting vascular fronts of P8 retinas. The graphs show quantification of the number $(n=24)$ and length $(n=120)$ of filopodia in tip endothelial cells (ECs). (D) IHC for CD31 and EC-specific molecule 1 (Esm1) in P5 retinas. (E) Labeling for CD31, ERG1, and EdU in the central areas of P8 retinas. The graphs show quantification of EC proliferation ( $n=24$ areas) and aneurysm ( $n=10$ retinas). ND, not detected. (F) 3D images of retinas labeled for CD31 and ERG1. See also Supplemental Video 1. (C) IHC for CD31 and cleaved caspase-3 in P8 retinas. The graph shows quantification of EC apoptosis ( $n=16$ areas). (H) P8 retina cups 24 hours after Evans Blue injections. (I) IHC for CD31, Ter-119, and fibrinogen in P8 retinas. (J) IHC for CD31 and biotin in P8 retinas after biotin perfusion. Note the focal biotin leakage (arrowheads). (K) Retina cups at P10. (L) H\&E staining of paraffin sections of P10 retinas shown in K. Right panels show magnified views of the boxed areas in the left panels. (M) IHC for CD31 and fibrinogen with nuclear labeling in cryosections of P10 retinas. Note the defects in vertical vascular sprouting, and fibrinogen deposition over the ganglion cell layer (GCL) and within the inner nuclear layer (INL) of the APB5-treated retina. ONL, outer nuclear layer. (N) IHC for vascular endothelial cadherin (VE-cad), ERG1, and claudin-5 in P8 retinal veins. (0) IHC for CD31, plasmalemma vesicle-associated protein (PLVAP), and NG2 in P8 retinas. (P) Transmission electron microscopy of P8 retinas after biotin perfusion. Note the deposition of electron dense tracers in disrupted EC junctions (arrowheads) and intracellular vesicles (arrow). Asterisks indicate vessel lumen. ${ }^{*} P<$ $0.05,{ }^{* *} P<0.01,{ }^{* * *} P<0.001$ (1-way ANOVA in B; 2-tailed Student's $t$ test in C, E, and G). Scale bars: $500 \mu \mathrm{m}$ (L, left); $50 \mu \mathrm{m}(\mathbf{A}, \mathbf{D}, \mathbf{G}, \mathbf{I}, \mathbf{J}, \mathbf{L}$ right, M, and 0); $20 \mu \mathrm{m}$ (C, E, and $\mathbf{F}) ; 5 \mu \mathrm{m}$ (N and $\mathbf{P})$.

decreased (Figure 2I and Supplemental Figure 2B), with a disappearance of opsin expression (Supplemental Figure 2C). The resulting vision impairment of the APB5-treated mice was documented by electroretinograms (Figure 2, J and K). After injecting $50 \mu \mathrm{g}$ of APB5, which induced retinal collapse at P10, the retinal tissue architecture was destroyed with subsequent blindness at 4 weeks (Figure 2, B, D, F, H, and J), reminiscent of end-stage DR. Although the severities of these phenotypes were almost equivalent in both eyes of most mice, we occasionally found remarkable retinal hemorrhage and edema in one eye, but not in the other (Figure 2L), as was the case in DR. In addition, we often found progressive disease exacerbation in the form of increased exudates and the expansion of edematous retinal areas (Figure 2M). Thus, our adult mouse model reproduced the characteristics of DR, with sustained vascular abnormalities and autonomous disease progression.

Irreversible BRB breakdown after transient $P C$ depletion. Despite the remaining vascular abnormalities, PCs existed in 4-week retinas after the APB5 injections at P1 (Figure 3A). However, the PC coverage was discontinuous in all types of retinal vessels, with significant vessel enlargements (Figure 3, A and B). Notably, aSMA expression was upregulated in venous and capillary PCs (Figure 3, C and D), suggesting the acquisition of myofibroblast properties (1). Moreover, PCs were often partially or completely dissociated from ECs (Figure 3, C and E). In these retinas, ECs still displayed round and enlarged cell shapes (Supplemental Figure 2D), and maintained angiogenic activities as shown by their proliferation and filopodia projections (Figure $3 \mathrm{~F}$ ). In contrast, we frequently detected apoptotic ECs, along with ghost vessels labeled by basement membrane sleeves without EC linings (28) (Figure 3G). Consistent with these results, serial angiography of the same retinal areas showed continuous vascular remodeling in the APB5-treated mice, without resolution of the retinal nonperfusion (Figure $3 \mathrm{H}$ ). These retinal vessels were highly leaky (Figure 3I and Supplemental Figure 2E), with claudin-5 downregulation and PLVAP upregulation (Figure 3, J and $\mathrm{K})$. Electron microscopy showed disrupted intercellular junctions and accelerated transcytosis in these ECs (Figure 3L). Together, these results indicated that transient failure of PC recruitment to growing retinal vessels resulted in irreversible defects in EC-PC associations and BRB breakdown.

Sustained inflammation in PC-free retinas. We then attempted to determine the sequential molecular and cellular events triggered by PC depletion. To identify gene expression changes in ECs completely devoid of PCs, we injected Tie2-GFP Tg mice (29) with $100 \mu \mathrm{g}$ of APB5 daily from P0, and performed microarray transcriptome analyses using $\mathrm{GFP}^{+}$ECs purified from P8 retinas by FACS (30). Compared with the control ECs, numerous genes were upregulated and downregulated in PC-free ECs, especially those encoding transporters and channels (Supplemental Table 1), which might contribute to barrier dysfunction. Furthermore, a series of inflammatory genes were significantly upregulated in PC-free ECs (Table 1), which was corroborated by the villous protrusions of vessel lumens (Supplemental Figure 1D), a characteristic feature in inflammatory ECs (27). Consistent with these observations, retinas of the DSCR-1-lacZ-Hprt reporter mice (31) treated with APB5 showed endothelial activation of the nuclear factor of activated T cells (NFAT) (Figure 4A), which targets most of the proinflammatory genes upregulated in PC-free ECs, including those encoding CCL2, VCAM-1, Angpt2, COX-2, CSF2, and E-selectin $(32,33)$. Notably, blockade of the NFAT pathway with cyclosporine suppressed retinal edema and hemorrhage with normalization of the vascular networks (Figure $4 \mathrm{~B}$ ), indicating the importance of inflammation in the BRB breakdown in PC-free vessels. In addition to ECs, 
A

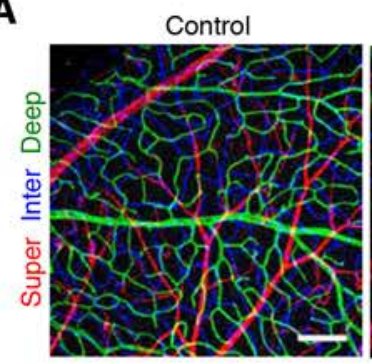

B
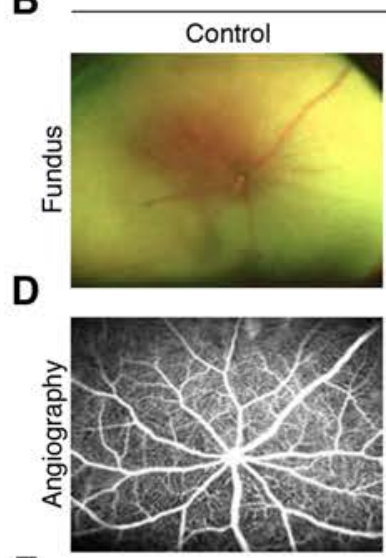

$\mathbf{F}$

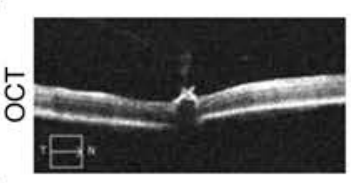

H

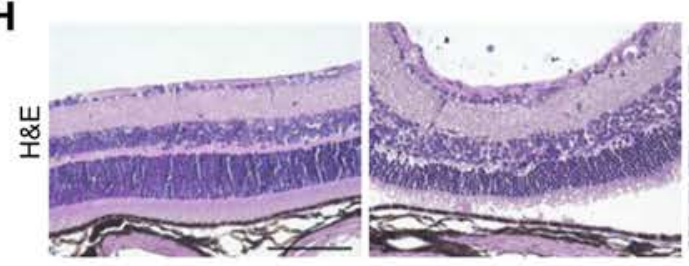

J

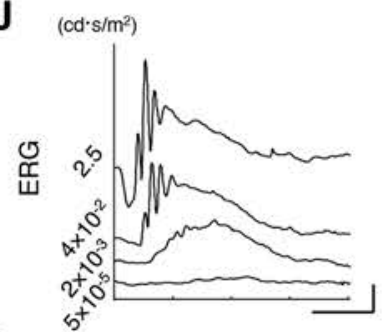

L
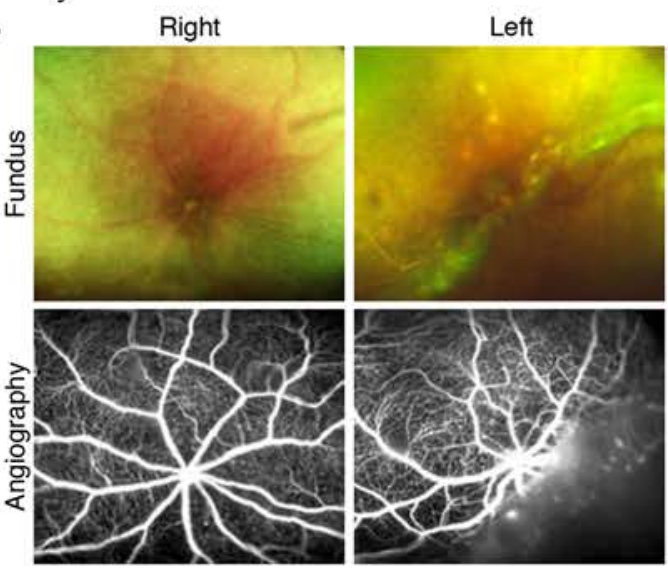

APB5 $30 \mu \mathrm{g}$

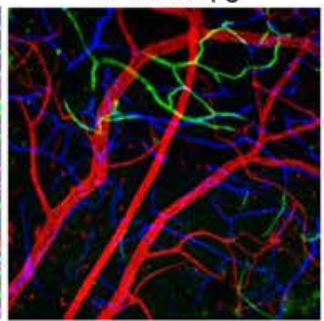

Mouse
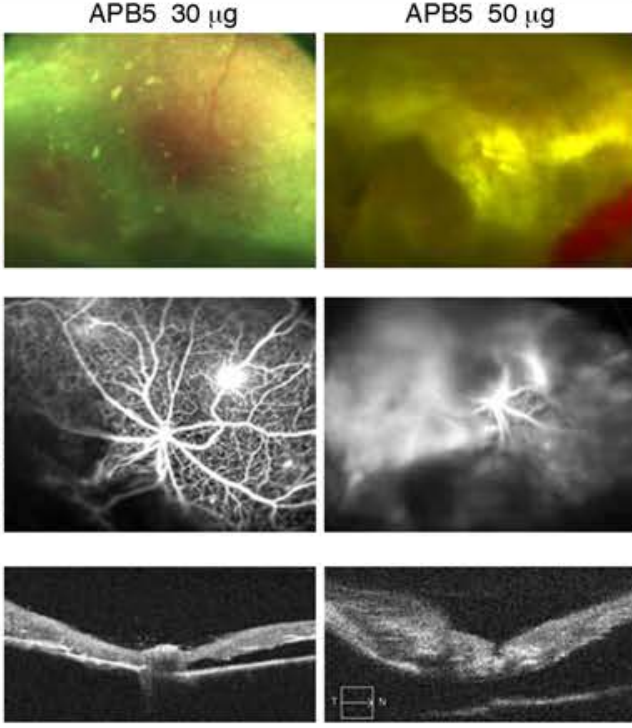

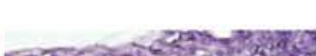
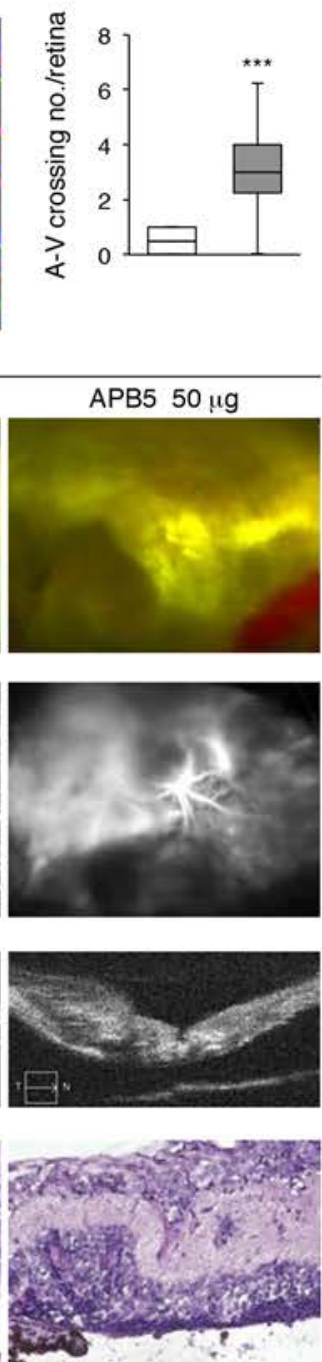

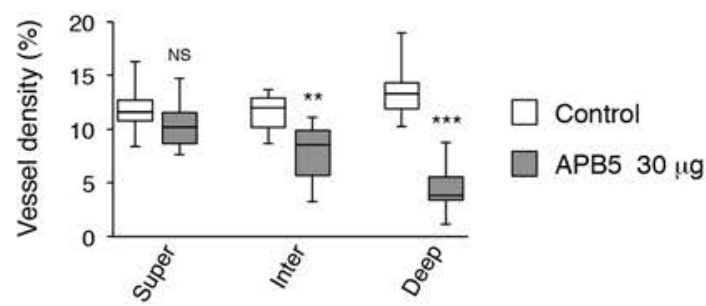

C

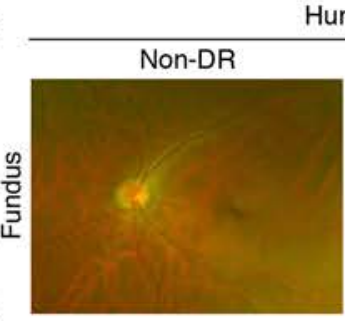

Human

E

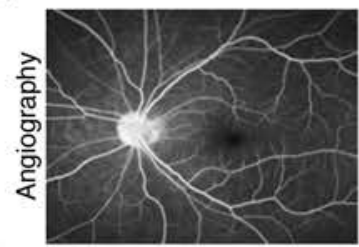

G

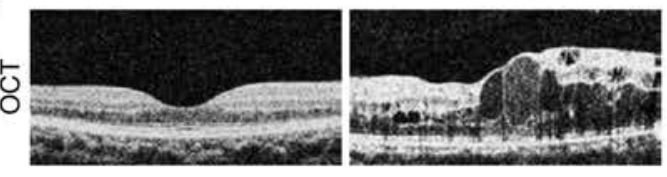

I

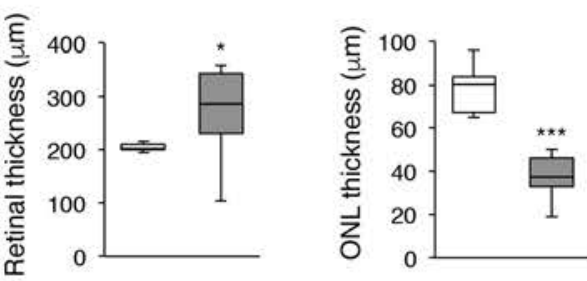

K

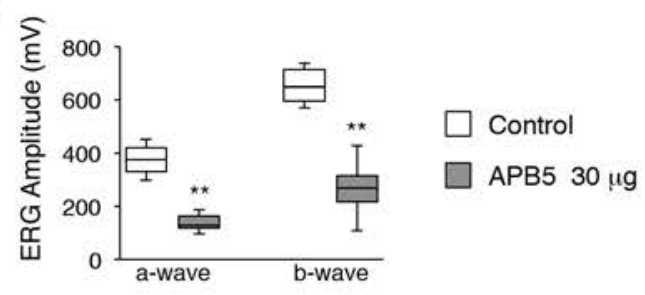

M

P21
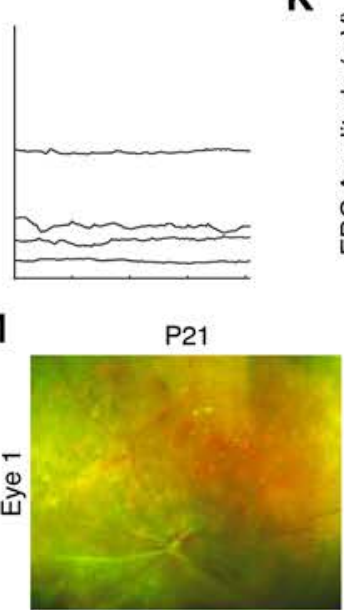

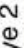

状

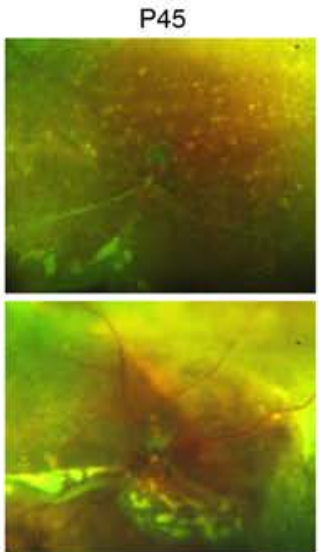

P70

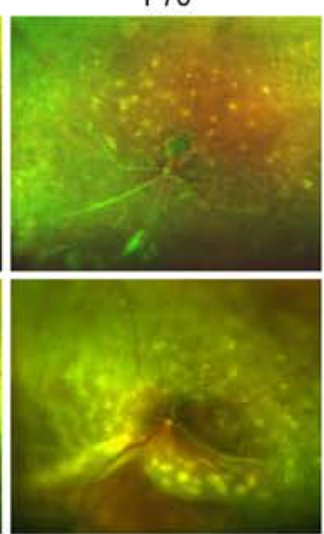


Figure 2. Adult mouse model reproducing characteristics of diabetic retinopathy (DR). (A) IHC for CD31 in 4-week retinas. Each vascular layer is shown in different colors. The graphs show quantification of artery-vein (A-V) crossings in the superficial vascular layer per retina $(n=10)$ and vessel density in each vascular layer ( $n=10$ areas). (B-C) Retinal images of 4 -week mice (B, D, and $\mathbf{F}$ ), and in humans (C, E, and $\mathbf{G})$. Note the similarities between APB5-treated retinas and human DR, such as yellow exudates (color fundus images in $\mathbf{B}$ and $\mathbf{C}$ ), vascular leakage and nonperfusion (fluorescein angiography in $\mathbf{D}$ and $\mathbf{E}$ ), and retinal edema and detachments (optical coherence tomography [OCT] images in $\mathbf{F}$ and $\mathbf{G}$ ). (H) H\&E staining in paraffin sections of 4-week mouse retinas. (I) Quantification of the thickness of retinas and the outer nuclear layer (ONL) measured by OCT $(n=10)$. See also Supplemental Figure 2B. (J) Scotopic full-field electroretinogram (ERG). (K) Quantification of the ERG amplitude of a-waves and b-waves ( $n=4$ mice). The results in B-H and J in mice and humans were obtained from identical eyes. (L) Color fundus photography and fluorescein angiography of both eyes from a 4-week mouse after injecting $30 \mu \mathrm{g}$ of APB5. Note the retinal exudates and edema in the left eye, and their absence in the right eye. (M) Time course of the individual eyes after injecting $30 \mu g$ of APB5. Note the increase in the number of retinal exudates in eyes 1 and 2 , and the expansion of retinal edema or detachment areas in eye $2 .{ }^{*} P<0.05,{ }^{*} P<0.01$, ${ }^{* * *} P<0.001$ (2-tailed Student's $t$ test). Scale bars: $100 \mu \mathrm{m}$ ( $\mathbf{A}$ and $\left.\mathbf{H}\right) ; 50 \mu \mathrm{s}$ for the horizontal line and $200 \mathrm{mV}$ for the vertical line (J).

proinflammatory genes such as $\operatorname{Tnf}, \mathrm{Il} 6$, and $C c l 2$ were continuously upregulated in whole retinas of both neonates and adults after APB5 injections (Figure 4C). Consistently, these retinas demonstrated increased infiltration of CD18+ leukocytes (Figure 4D) and endothelial upregulation of ICAM-1 (Figure 4E). To our surprise, a single injection of a small amount of APB5 induced sustained infiltration of leukocytes labeled by acridine orange (34), even without retinal edema and hemorrhage (Figure 4, F and G), suggesting that inflammation can serve as a signature of $\mathrm{PC}$ loss in retinas.

$M P$ infiltration in $P C$-free retinas. To determine the leukocyte subpopulations infiltrating in PC-free retinas, we performed flow cytometry analyses in P8 retinas (Supplemental Figure 3A), by combining the pan-leukocyte marker CD45 with the myeloid marker CD11b. Compared with the controls, APB5 injections resulted in a more than 10 -fold increase of the $\mathrm{CD} 45^{\mathrm{hi}} \mathrm{CD} 11 \mathrm{~b}^{+}$cell fraction (Figure $5 \mathrm{~A}$ ), which might represent monocyte-derived MPs (35). Indeed, more than $85 \%$ of this population expressed the monocyte/ MP marker, Ly-6C, whereas less than $10 \%$ was positive for the granulocyte marker, Gr-1 (Figure 5A). The APB5-treated retinas showed a 6.7 -fold increase of $\mathrm{CD} 45^{\mathrm{hi}} \mathrm{CD} 11 \mathrm{~b}+\mathrm{Ly}-6 \mathrm{C}^{+}$cells over those of the control (Figure 5A). In contrast, we found no significant changes in the number of $\mathrm{CD}^{2} 5^{1 \circ} \mathrm{CD} 11 \mathrm{~b}^{+} \mathrm{Ly}-6 \mathrm{C}^{+}$cells, which might represent resident microglia (35). Notably, the Cx3cr1-GFP reporter mice (36), in which GFP expression coincided with various MP markers (Supplemental Figure 3B), showed increased infiltration of perivascular MPs at P6 in APB5-treated retinas (Supplemental Figure 3C), which was sustained even at 4 weeks (Figure 5B). Electron microscopy further demonstrated direct EC-MP contacts in PC-free retinal vessels (Figure $5 \mathrm{C}$ ). In contrast to the resident microglia displaying a ramified configuration with extensive processes, the infiltrating MPs displayed round and enlarged amoeboid cell bodies with shortened dendrites (Figure 5, B and D). Furthermore, real-time imaging of mouse retinas showed decreased dendrite motility in these MPs both at P8 and 4 weeks (Figure 5E, and Supplemental Videos 2 and 3). These results suggested mutual communications between activated MPs and denuded ECs. Intriguingly, MP depletion with clodronate liposomes after APB5 injections dramatically suppressed retinal edema and hemorrhage, with restoration of vessel organization (Figure $5 \mathrm{~F}$ ), indicating the role of MPs in damaging PC-free vessels.

EC-MP interactions via VEGF and PlGF in PC-free retinas. In APB5-treated retinas, sustained hypoxia along with vessel hypoperfusion (Figure 6A) suggested the upregulation of hypoxia-inducible genes. To identify distinct MP-derived signals affecting the EC integrity, we first focused on VEGF, which was continuously upregulated in APB5-treated retinas (Figure 6B). During retinal vascular development, the Vegfa

Table 1. Inflammatory genes upregulated in pericyte-free endothelial cells

\begin{tabular}{|c|c|c|c|c|}
\hline Probe set ID & Gene symbol & Gene title & Fold change & Local FDR \\
\hline 102736_at & $\mathrm{Cc} / 2$ & chemokine (C-C motif) ligand 2 & 5.94 & 0.044 \\
\hline 92558_at & Vcam1 & vascular cell adhesion molecule 1 & 3.12 & 0.027 \\
\hline 92210_at & Angpt2 & angiopoietin 2 & 2.85 & 0 \\
\hline 104647_at & Ptgs2 & prostaglandin-endoperoxide synthase 2 & 2.45 & 0.118 \\
\hline 92948_at & {$[s f 2$} & colony stimulating factor 2 & 2.44 & 0.03 \\
\hline 98811_at & Ptgir & prostaglandin I receptor & 2.29 & 0.022 \\
\hline 99333_at & Sele & selectin, endothelial cell & 2.17 & 0.235 \\
\hline
\end{tabular}

Probe sets enriched more than 2-fold in retinal GFP+ cells sorted from P8 Tie2-GFP Tg mice after daily APB5 injections compared with those after PBS injections. Data were obtained from 3 independent experiments. 
A
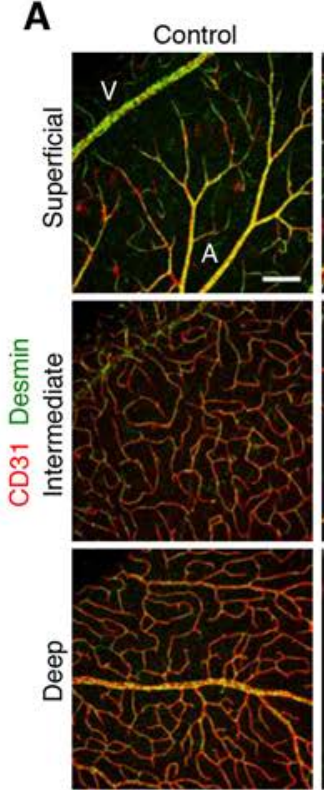

F
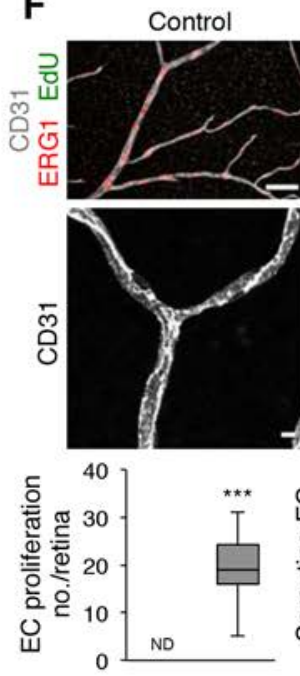

APB5 $30 \mu \mathrm{g}$
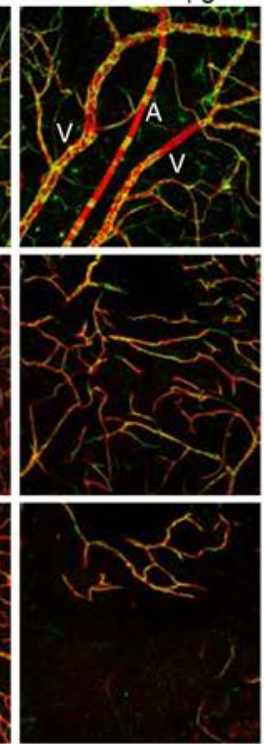

APB5 $30 \mu \mathrm{g}$
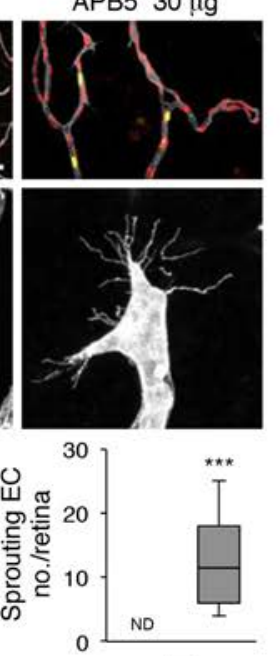

B
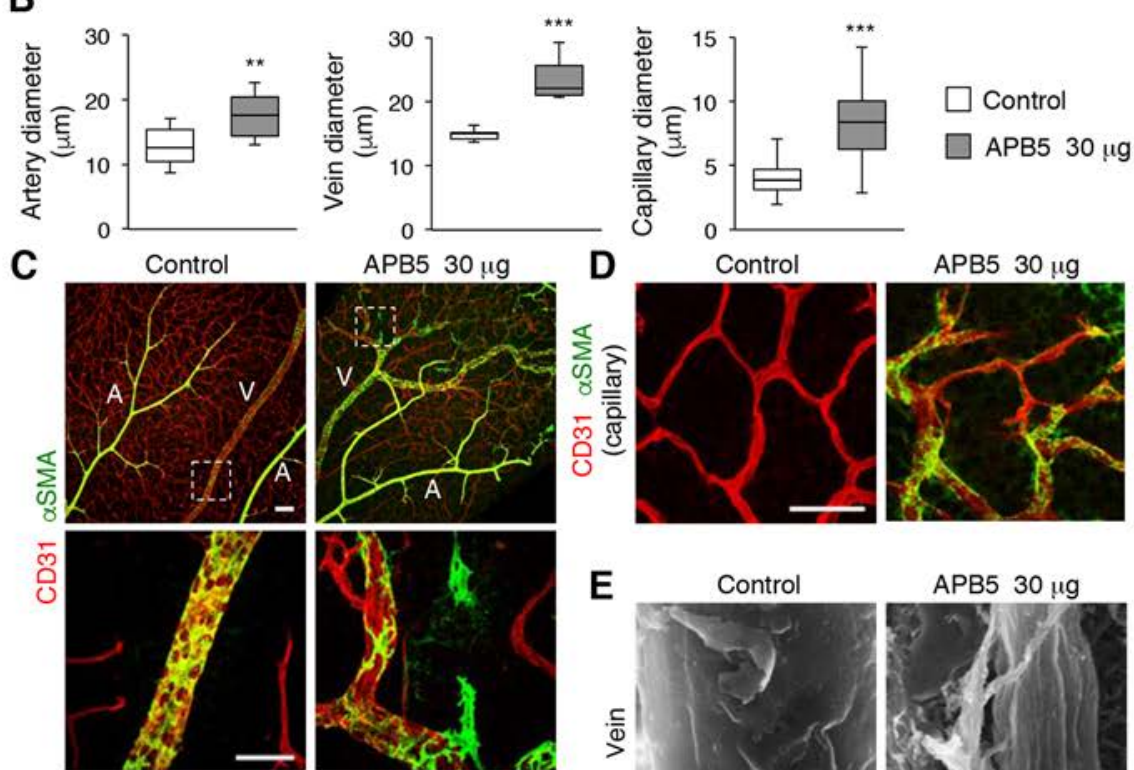

G
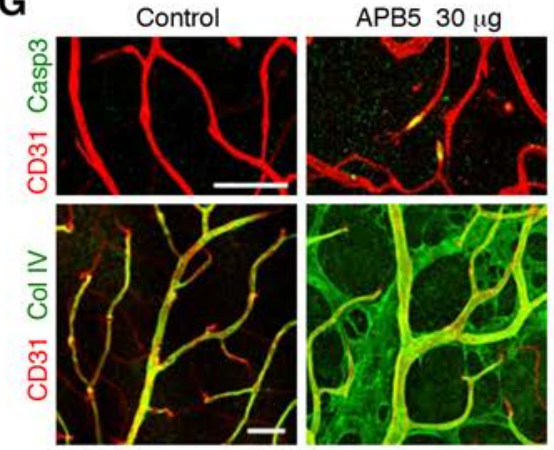

E

당

Control

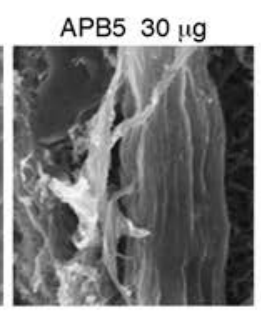

H
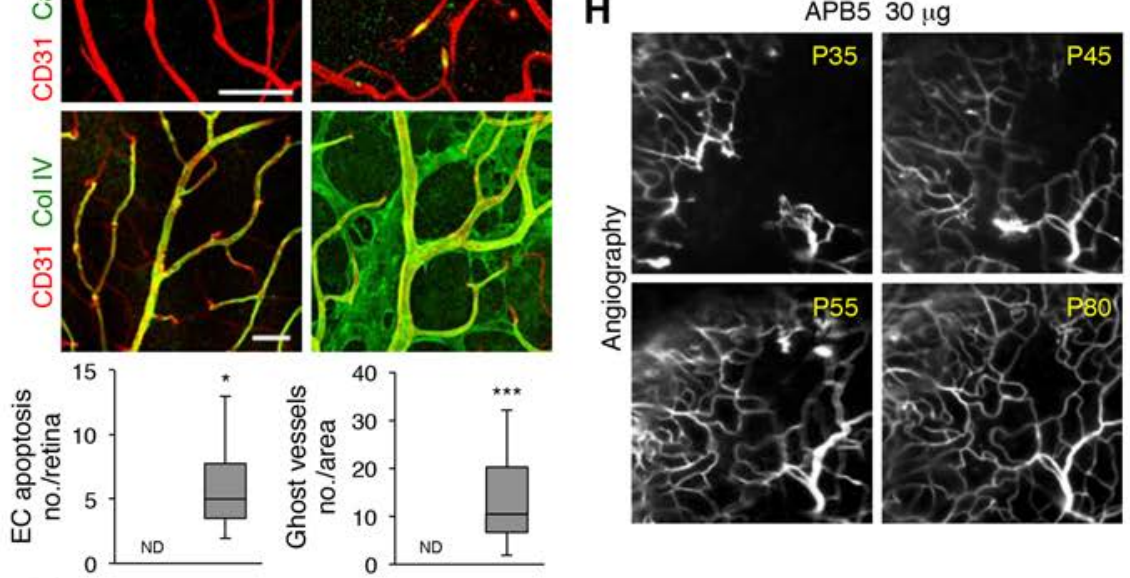

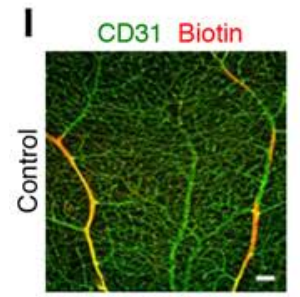

J VE-cad ERG1
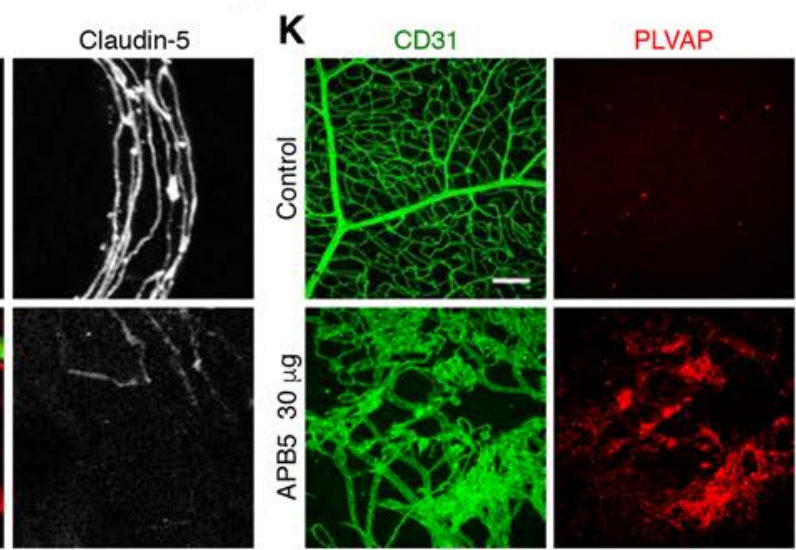

$\mathbf{L}$
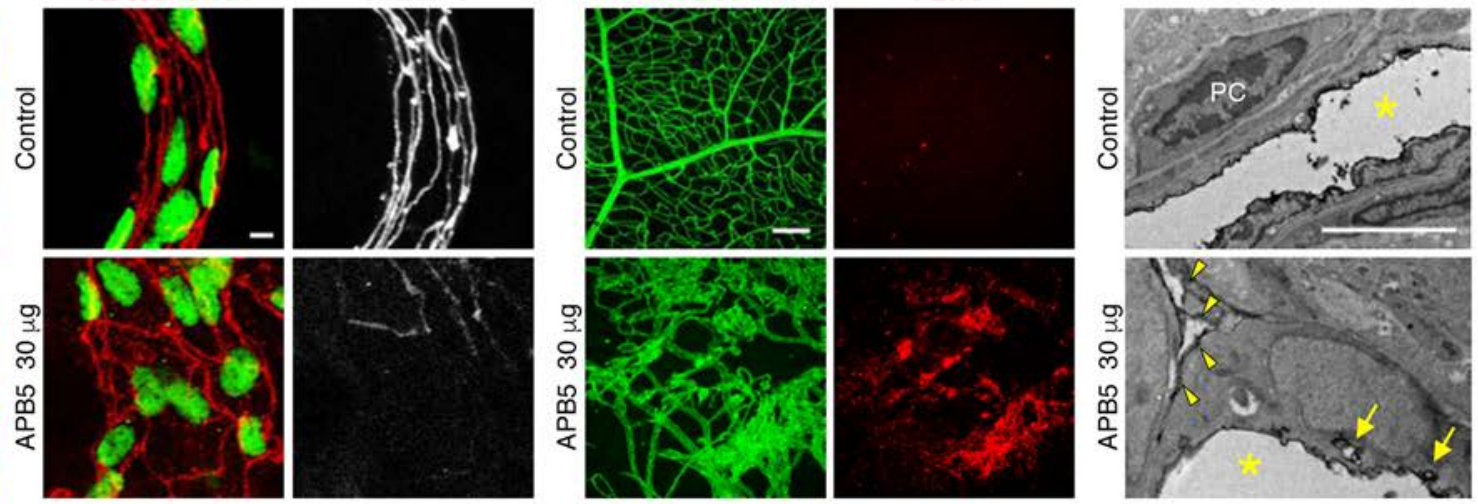

Figure 3. Irreversible retinal vascular dysfunctions after transient PDGFR $\beta$ inhibition. (A) IHC for CD31 and desmin in the 3 vascular layers of 4-week retinas (related to Figure 2A). (B) Quantification of diameters of arteries $(n=10)$, veins $(n=10)$, and capillaries $(n=50)$ in the superficial vessels of 4-week retinas. (C and D) IHC for CD31 and $\alpha$-smooth muscle actin ( $\alpha S M A$ ) in 4-week retinas. Lower panels in $\mathbf{C}$ are magnified views of the boxed areas (veins) in the upper panels. Note the $\alpha$ SMA upregulation in venous and capillary pericytes (PCs), and their dissociation from the vessel walls in the APB5-treated retinas. (E) Scanning electron microscopy of 4-week retinal veins. (F) Labeling for CD31, Ets-related gene-1 (ERG1), and 5-ethynyl-2'-deoxyuridine (EdU, upper), and for CD31 (lower) in 4-week retinas. The graphs show the number of proliferating endothelial cells (ECs) per retina (control, $n=6 ; \mathrm{APB} 5, n=8$ ) 
and sprouting ECs with filopodia projections per retina $(n=10)$. (G) IHC for CD31 combined with cleaved caspase-3 (upper) or type IV collagen (Col IV; lower) in 4-week retinas. Note the ghost vessels represented by basement membrane sleeves without EC linings in the APB5-treated retina. The graphs show the number of apoptotic ECs per retina $(n=4)$ and ghost vessels per area $(n=16)$. (H) Sequential monitoring of fluorescein angiography in the same retinal area. Note the continuous vascular remodeling without resolving nonperfusion. (I) IHC for CD31 and biotin after biotin perfusion in 4-week retinas. (J) IHC for vascular endothelial cadherin (VE-cad), ERG1, and claudin-5 in 4-week retinal veins. (K) IHC for CD31 and plasmalemma vesicle-associated protein (PLVAP) in 4-week retinas. (L) Transmission electron microscopy after biotin perfusion in 4-week retinas. Note the deposition of electron-dense tracers in disrupted EC junctions (arrowheads) and intracellular vesicles (arrows). Asterisks indicate vessel lumen. ${ }^{*} P<0.05,{ }^{* *} P<0.01,{ }^{* *} P<0.001$ (2-tailed Student's $t$ test). Scale bars: $100 \mu \mathrm{m}$ (A, C upper, I, and $\mathbf{K}$ ); $50 \mu \mathrm{m}$ (C lower, D, $\mathbf{F}$ upper, and $\mathbf{C}) ; 5 \mu \mathrm{m}$ (E, F lower, J, and L).

gene is expressed predominantly in astrocytes, but not in resident microglia, whereas the Vegfr2 gene is expressed in angiogenic ECs as well as in neurons and Müller glia (26, 37). However, the endothelial VEGFR2 protein is barely detectable because of its rapid turnover (38). In contrast to these normal situations, perivascular MPs abundantly expressed Vegfa in the APB5-treated retinas (Figure 6C). Furthermore, PC-free ECs highly upregulated VEGFR2 both at the gene and protein expression levels, as demonstrated in Vegfr2-BAC-GFP reporter mice (39) (Figure 6D). This endothelial VEGFR2 upregulation was maintained even at 4 weeks in the APB5-treated retinas (Figure 6D). Together, these results suggested that MP-derived VEGF constantly activated the endothelial VEGFR2 in PC-free retinal vessels.

We next investigated the involvement of P1GF, which was 8.6-fold upregulated in 8-week retinas after APB5 injection (Figure 6B). Similar to Vegfa, perivascular MPs strongly expressed Pgf in P8 APB5-treated retinas, contrasting with its absence in resident microglia in the controls (Figure 6E). Furthermore, Vegfr 1 promoter activation, which was restricted to ECs in the controls, was continuously upregulated in retinal MPs in the APB5-treated Vegfr1-BAC-DsRed reporter mice (40) (Figure 6F). Flow cytometry analyses further demonstrated that more than $90 \%$ of the CD $45^{\text {hi }} \mathrm{CD} 11 \mathrm{~b}^{+}$cells expressed VEGFR1 in the P8 APB5treated retinas, with a 6.3 -fold increase in the number of $C D 45^{\text {hi }} \mathrm{CD} 11 \mathrm{~b}^{+} \mathrm{VEGFR} 1^{+}$cells compared with that of the controls (Figure 6G). To assess the function of the VEGFR1 signal in the PC-free retinal vessels, we analyzed Flt1-TK ${ }^{-/}$mice which expressed a mutant form of VEGFR1 lacking the cytoplasmic tyrosine kinase domain (41). After injecting the control PBS, the Flt1-TK $\mathrm{K}^{-1-}$ mice displayed no obvious abnormalities in retinal vascular formation, excluding the effect of VEGFR1 inactivation on retinal ECs and resident microglia (Figure 6H). In contrast, after APB5 injection, the Flt1-TK $K^{-1-}$ mice showed dramatic resolution of vascular abnormalities with a significant decrease in MP infiltration (Figure $6 \mathrm{H}$ ). Furthermore, the ex vivo retina imaging showed that sequestration of VEGF and PIGF by VEGF Trap (42) significantly reduced MP motility with recovery of dendrite elongation in APB5-treated retinas (Figure 6I and Supplemental Video 4). These results suggested that MP-derived VEGF and/or P1GF activated VEGFR1 in MPs in an autocrine or paracrine manner, thereby promoting MP migration and disease exacerbation in PC-free retinas.

Angpt2 upregulation and FOXO1 activation in PC-free ECs. To further identify the EC-derived signals acting on ECs or MPs, we focused on Angpt2, which was 2.9-fold upregulated in PC-free ECs (Table 1). While the Angpt2 gene was specifically expressed in tip ECs during retinal vascular development (43), its transcription was highly upregulated in stalk ECs at P4 after APB5 injection (Figure 7A). IHC analyses further revealed the complementary Angpt2 upregulation and Tie2 downregulation in PC-free ECs (Figure 7B). This Angpt2 upregulation was sustained in adult retinas even after a single APB5 injection (Figure 7, B and C). Although Tie2 is reportedly expressed in a subset of MPs in several pathophysiological settings (44), its expression was confined to ECs in our model (Figure 7, D and E). In order to assess the activation of Tie2 in these settings, we performed immunoprecipitation and immunoblotting experiments using whole lysates of P8 retinas. Unexpectedly, the overall Tie2 phosphorylation levels were significantly increased in APB5-treated retinas (Figure 7F). Nonetheless, at the aneurysm-like structures, we found the nuclear translocation of FOXO1 with intense Angpt2 expression in ECs, suggesting the local, but not uniform, Tie2 inactivation in PC-free retinal vessels (Figure 7, G and $\mathrm{H}$ ). Consistent with these observations, the endothelial immunoreactivity for Tie1, but not Tie2, was faint in the APB5-treated retinas, despite the unaltered expression levels of the Tie1 gene (Figure 7, I and J). Together with the expression of VEGF and TNF- $\alpha$ in perivascular MPs (Figures $6 \mathrm{C}$ and $7 \mathrm{~K}$ ), our results suggested that MPs facilitated Tie1 cleavage in PC-free ECs, thereby converting EC-derived Angpt2 into a Tie2 antagonist and activating FOXO1 in localized retinal areas, which might be responsible for the prominent vascular leakage from aneurysms.

Synergistic effects of blocking Angpt2, VEGF, and PlGF in vascular normalization. To evaluate the effects of neutralizing Angpt2, VEGF, and P1GF on PC-free retinal vessels, we intravitreally injected an anti-Angpt2 $\mathrm{mAb}$ (45) and VEGF Trap at P7 after i.p. injections of $50 \mu \mathrm{g}$ of APB5 at P1. At P13, gross retinal observations 
A

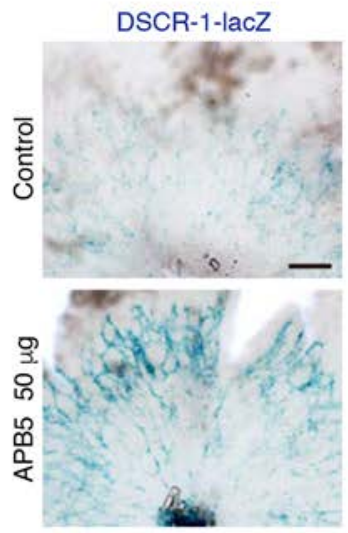

B

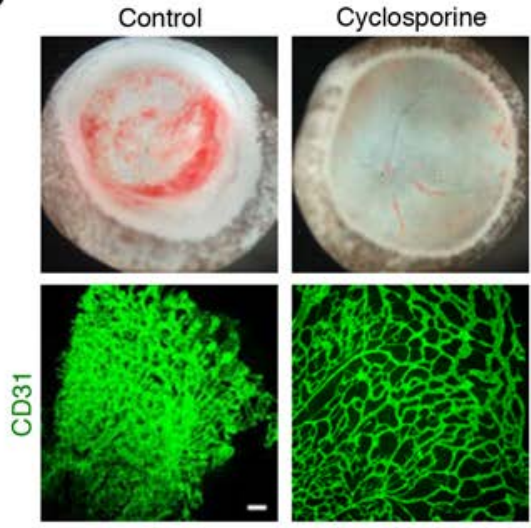

\begin{tabular}{ccc} 
Grade & Control & Cyclosporine \\
\hline 1 & $0(0 \%)$ & $3(22 \%)$ \\
2 & $0(0 \%)$ & $7(50 \%)$ \\
3 & $0(0 \%)$ & $2(14 \%)$ \\
4 & $14(100 \%)$ & $2(14 \%)$ \\
\hline
\end{tabular}

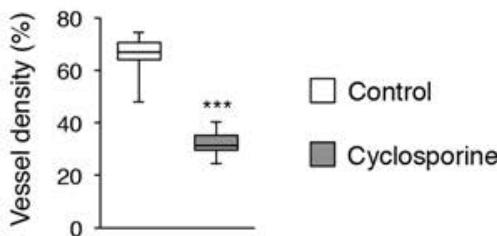

C

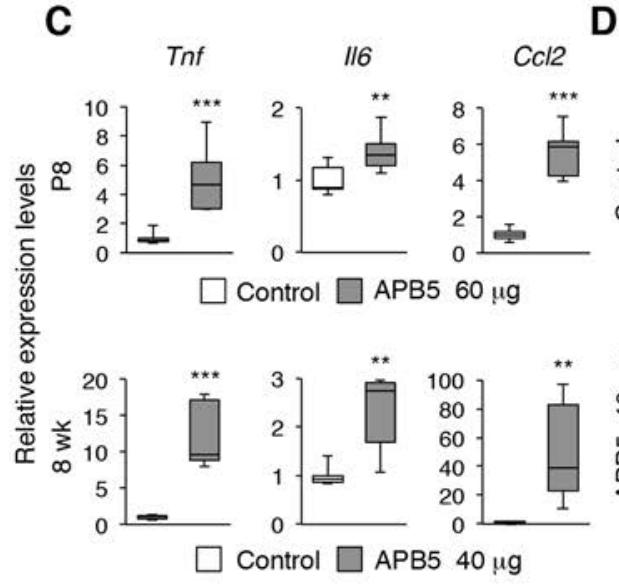

F
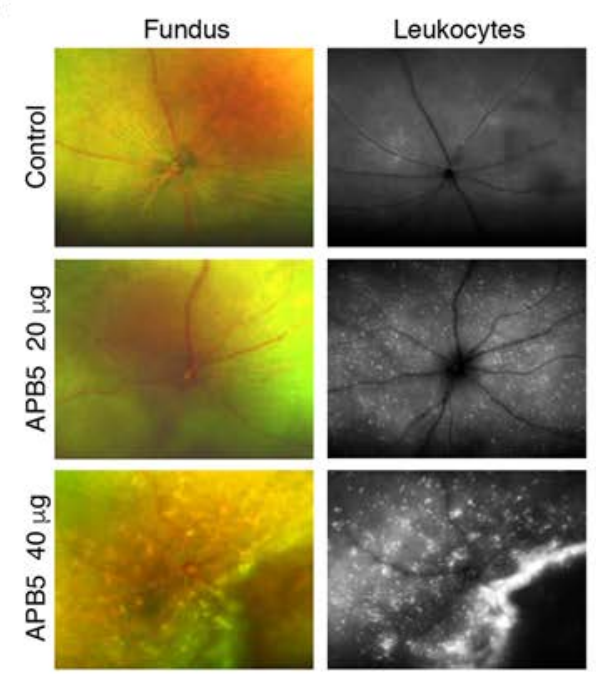

D<smiles>[Pb]C=[Pb]</smiles>
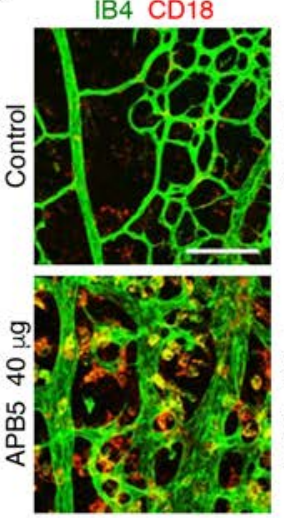

E
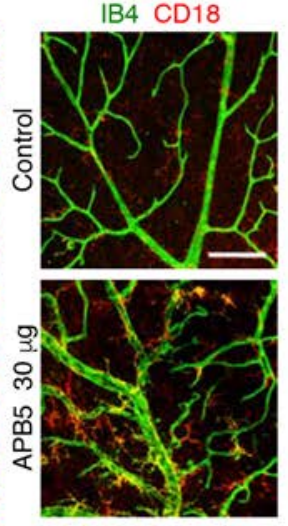

P8

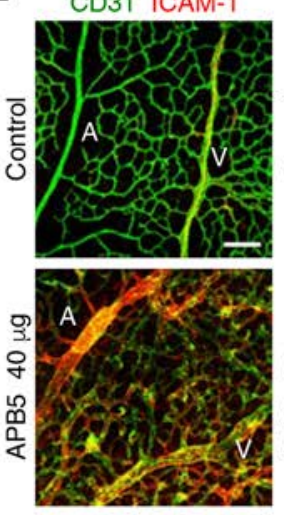

4 wk CD31 ICAM-1
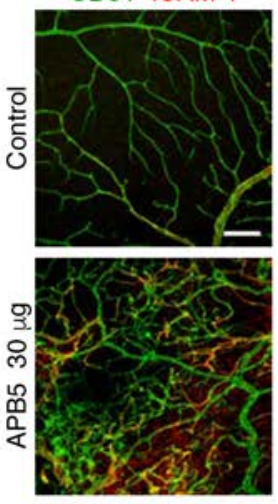

G
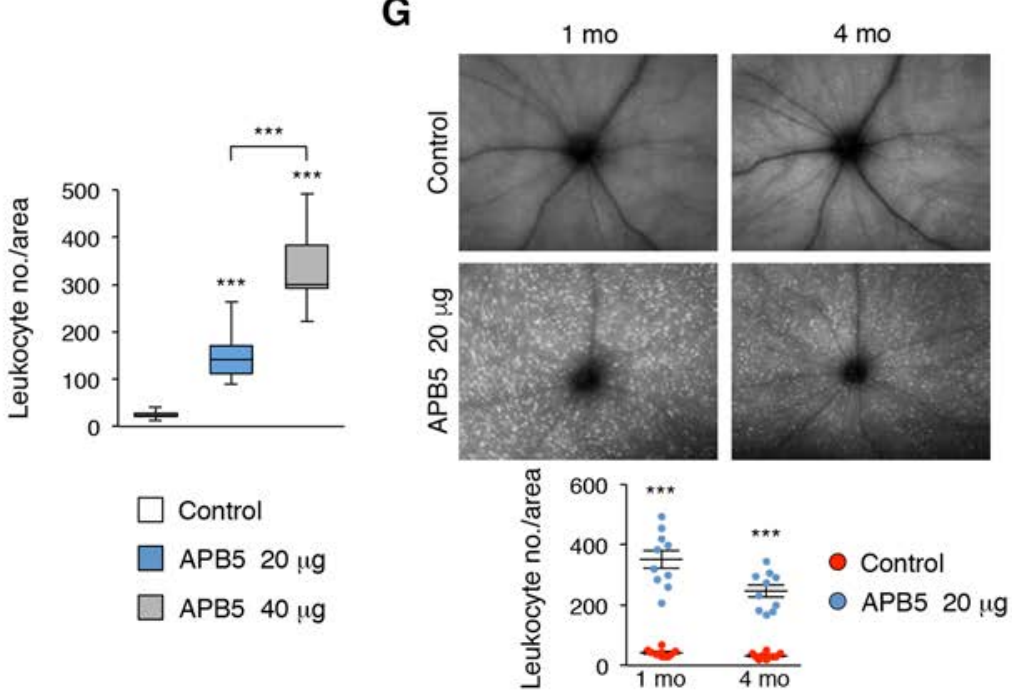

Figure 4. Sustained inflammation in pericyte-free retinas. (A) $\beta$-gal staining in retinas of P5 DSCR-1-lacZ-Hprt mice. (B) Representative retina cups (upper) and IHC for CD31 (lower) at P11 after injecting $50 \mu$ of APB5 at P1. Control vehicle or cyclosporine was i.p. injected at P7 and P8. Retinas were graded according to Supplemental Figure 1C. The graph shows the vessel density ( $n=20$ areas). (C) Quantitative reverse transcription PCR (qRT-PCR) of P8 $(n=8)$ and 8-week $(n=6)$ retinas. ( $\mathbf{D}$ and $\mathbf{E})$ Labeling for isolectin B4 (IB4) and CD18 (D), and CD31 and ICAM-1 (E). Note that the arterial ICAM-1 expression was undetectable in the control, but was upregulated in the APB5-treated retina. (F) Retina imaging showing color fundus photography (left) and leukocyte infiltration labeled by acridine orange (right) in 4-week retinas. The graph shows the number of leukocytes ( $n=12$ areas). (C) Time course of acridine orange labeling in the individual retinas. The graph shows the number of leukocytes $\left(n=10\right.$ areas). The horizontal lines represent the mean \pm SEM. ${ }^{* *} P<$ $0.01,{ }^{* *} P<0.001$ (2-tailed Student's $t$ test in B, C, and G; 1-way ANOVA in F). Scale bars: $200 \mu \mathrm{m}(\mathbf{A}) ; 100 \mu \mathrm{m}$ (B, D, and E). 
A
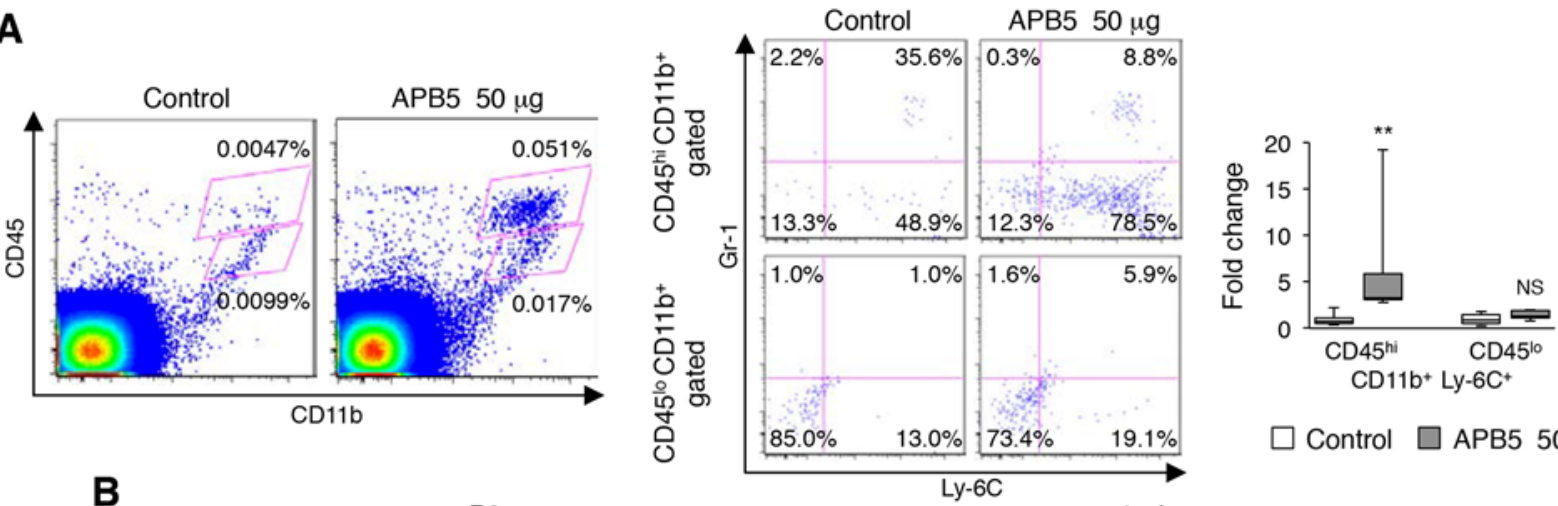

B

P8

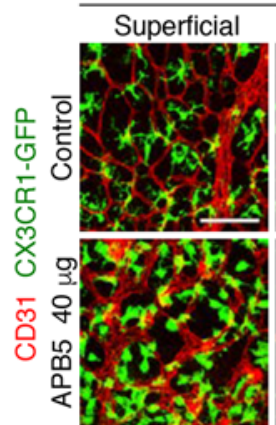

Intermediate

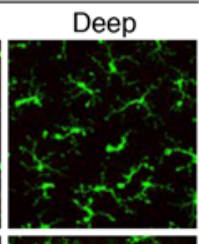

$\square$ Control $\square$ APB5 $50 \mu \mathrm{g}$
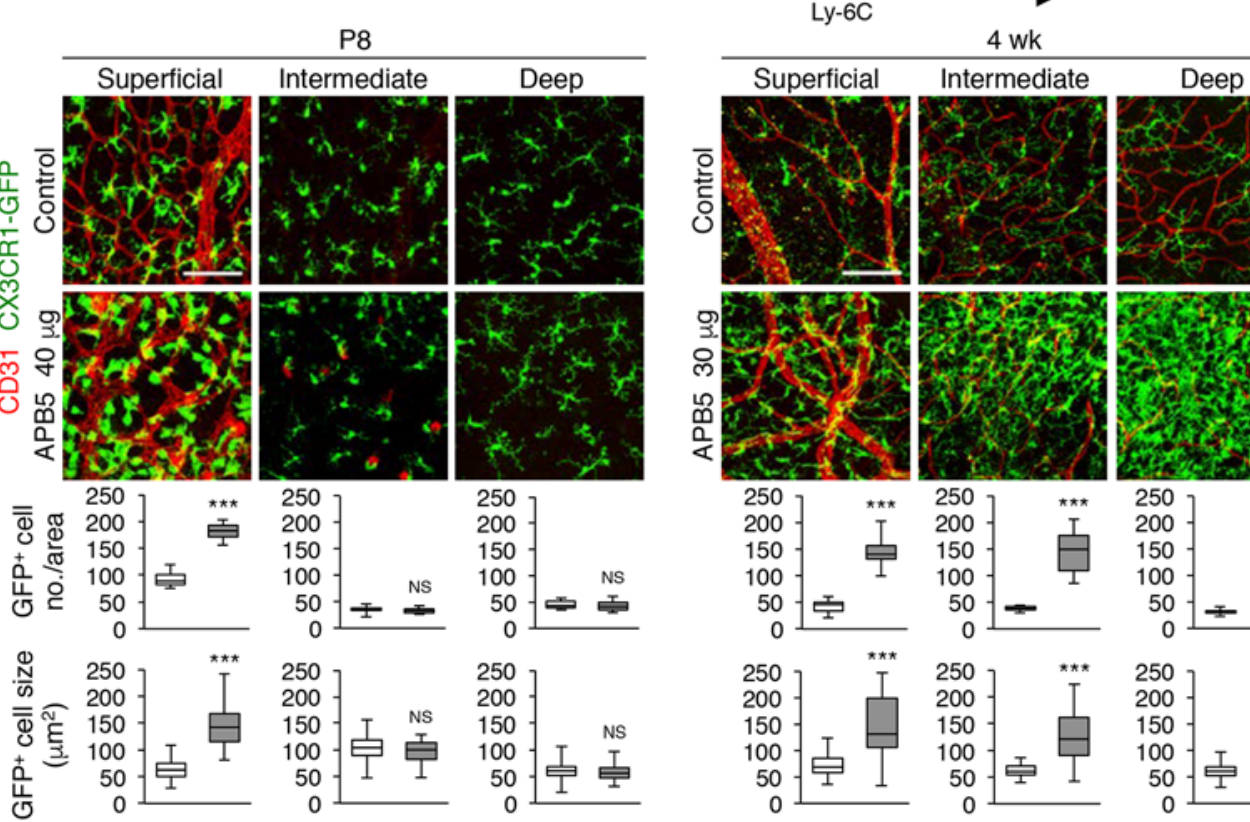

$\square$ Control $\square$ APB5 $40 \mu \mathrm{g}$

D

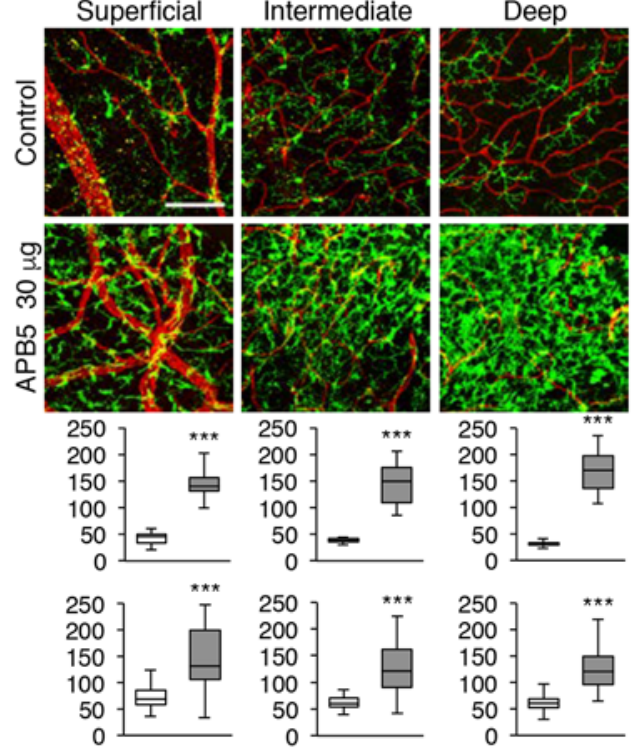

$\square$ Control $\square$ APB5 $30 \mu \mathrm{g}$
C
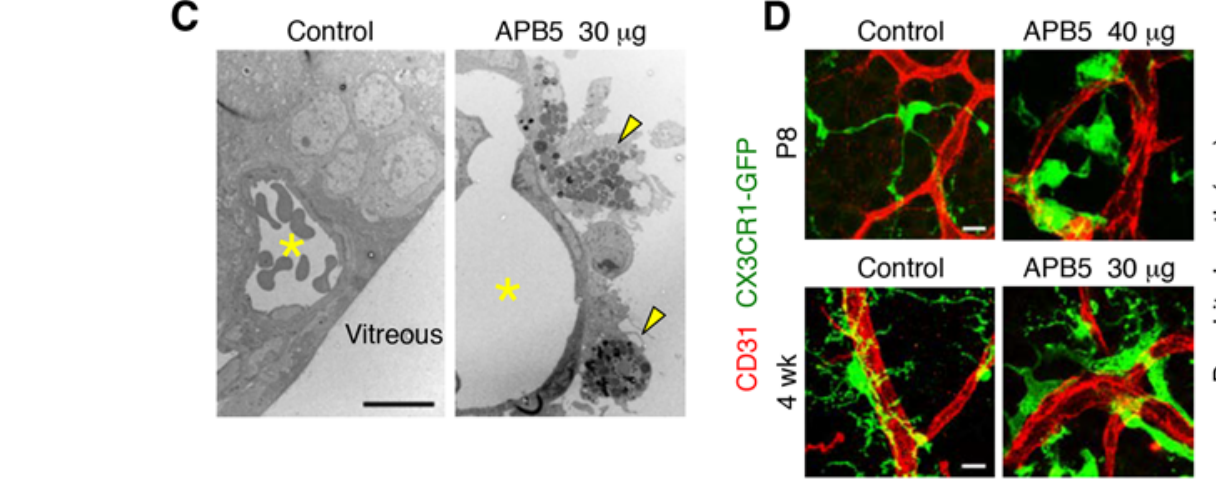

$\mathbf{F}$

F Control Clodronate

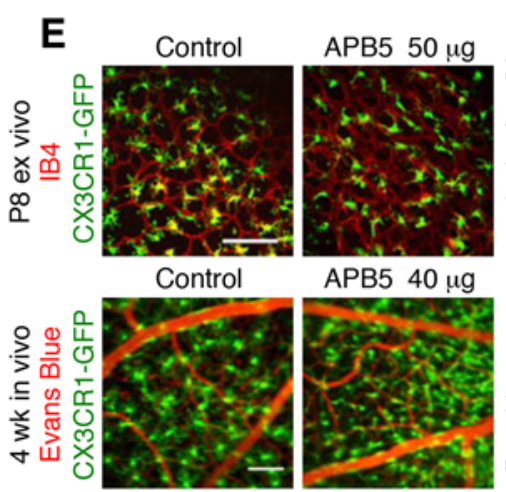

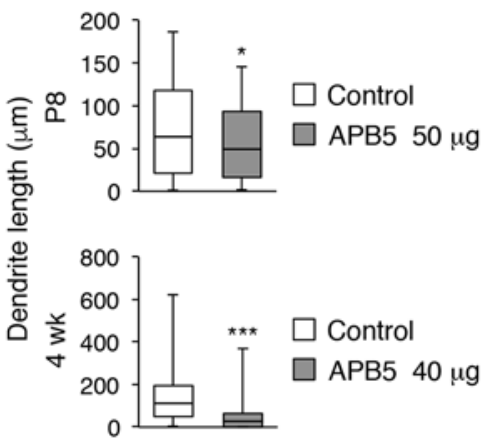

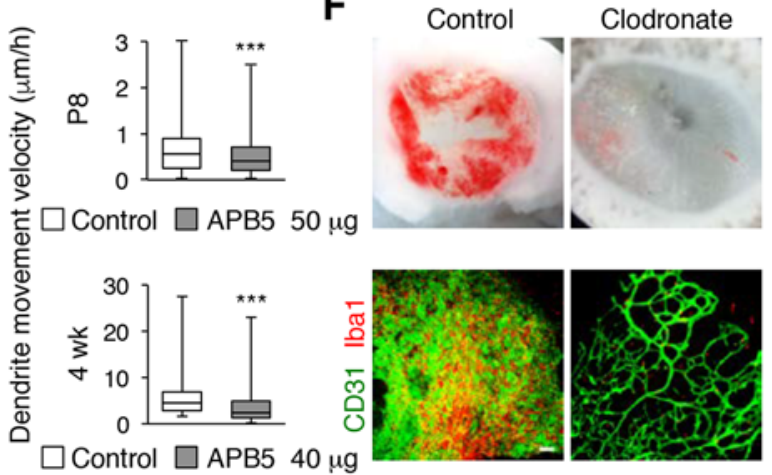

\begin{tabular}{|c|c|c|}
\hline Grade & Control & Clodronate \\
\hline 1 & $0(0 \%)$ & $12(100 \%)$ \\
\hline 2 & $0(0 \%)$ & $0(0 \%)$ \\
\hline 3 & $0(0 \%)$ & $0(0 \%)$ \\
\hline 4 & $12(100 \%)$ & $0(0 \%)$ \\
\hline 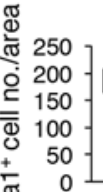 & 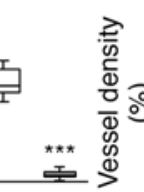 & $\left.\begin{array}{r}80 \\
60 \\
40 \\
20 \\
0\end{array}\right] . \begin{array}{ll} \\
\text { E* }\end{array}$ \\
\hline
\end{tabular}




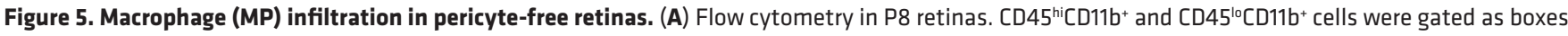
in the left panels. The graph shows the fold changes of the number of CD45 hiCD11b+Ly-6C+ and CD45 $5^{\circ} C D 11 b^{+} L y-6 C^{+}$cells per retina $(n=5)$. (B) Labeling for CD31 and GFP in retinas of CX3cr1-GFP mice. The graphs show the number of GFP+ cells per area $(P 8, n=20 ; 4$ week, $n=10)$ and their cell body size ( $n=50$ ). (C) Transmission electron microscopy of 4-week retinas without biotin perfusion. Note the direct contacts of MPs (arrowheads) with denuded endothelial cells in the APB5-treated retina. Asterisks indicate the vessel lumen. (D) Labeling for CD31 and GFP in retinas of Cx3cr1-GFP mice. The graphs show quantification of total dendrite length per GFP+ cell (P8 control, $n=77$; P8 APB5, $n=67$; 4-week control, $n=191 ; 4$-week APB5, $n=107$ ). (E) Photographs of ex vivo (upper) and intravital (lower) retina imaging from CX3cr7-GFP mice. The graphs show the dendrite movement velocity (P8 control, $n=185 ;$ P8 APB5, $n=435$; 4-week control, $n=816$; 4-week APB5, $n=796$ ). See also Supplemental Videos 2 and 3. (F) Representative retina cups (upper) and IHC for CD31 and ionized calcium binding adapter molecule 1 (lba1; lower) at P11 after injecting $50 \mu \mathrm{g}$ of APB5 at P1. Control liposomes or clodronate liposomes were i.p. injected at P7 and P8. Retinas were graded according to Supplemental Figure $1 \mathrm{C}$. The graphs show the number of Iba1 ${ }^{+}$cells and the vessel density per area (control, $n=20$; clodronate, $n=28$ ). ${ }^{*} P<0.05,{ }^{* *} P<0.01,{ }^{* * *} P<0.001$ (2-tailed Student's $t$ test). Scale bars: $100 \mu \mathrm{m}$ (B, E, and F); $10 \mu \mathrm{m}$ (D); $5 \mu \mathrm{m}$ (C).

showed that VEGF Trap was more effective than the anti-Angpt2 mAb in suppressing edema and hemorrhage, whereas a combination of these drugs exerted synergistic effects (Figure 8A). Their ability to restore the BRB integrity was further illustrated by decreased extravasations of red blood cells and fibrinogen exudates, with claudin-5 upregulation and PLVAP downregulation in ECs (Figure 8, B-E). These treatments also suppressed inflammation with reduced MP infiltration and endothelial ICAM-1 downregulation (Figure 8, B and F). Moreover, EC-PC associations were recovered with $\alpha$ SMA downregulation in PCs and restoration of EC shapes (Figure 8, B and G). Consequently, vascular patterning was ameliorated with the disappearance of aneurysm-like structures (Figure 8, B-G). These effects were sustained in 4-week retinas even after a single drug injection at P7 (Figure $8 \mathrm{H}$ ). Although intravitreal drug injections at 4 weeks failed to normalize the disturbed retinal structures induced by $50 \mu \mathrm{g}$ of APB5 (data not shown), the anti-Angpt $2 \mathrm{mAb}$ and VEGF Trap synergistically suppressed retinal inflammation induced by $20 \mu \mathrm{g}$ of APB5 (Supplemental Figure 4). Thus, simultaneous blockade of Angpt2, VEGF, and P1GF efficiently shut down the damaging cycle of inflammation and vascular dysfunctions caused by PC depletions in retinal vessels.

\section{Discussion}

In most diabetic patients, the early symptoms of retinopathy, such as microaneurysm formation with elevated vascular leakage, become clinically manifested many years after the hyperglycemic periods. To understand the crucial events during the long time course of DR, it is desirable to investigate an animal model in which direct ablations of specific cells or molecules can reproduce the characteristic features of DR. Here, we successfully established a mouse model mimicking various forms of DR, in which the disease severity in adult retinas corresponded to the extent of PC depletion from developing retinal vessels. Thus, our model may provide insight into the pathological changes that occur after PC depletion in human retinas. For example, PC-free retinal vessels formed aneurysm-like structures, in which actively proliferating ECs accumulated without properly oriented sprouting. Given the similarity of this morphological defect to that caused by an endothelial deficiency of serum response factor, a transcription factor downstream of the VEGF signal $(46,47)$, it is plausible that microaneurysm formation in DR may, at least in part, be a consequence of dysregulated EC proliferation in response to VEGF, rather than dilation of PC-free vessels. Furthermore, focal leakage from these aneurysms in our model suggested that the hyperpermeability in PC-free retinal vessels was attributable, not only to their mechanical fragility, but also to a local damaging cycle in ECs, as discussed below.

Although our model may represent essential aspects of disease progression arising from PC loss, it should be noted that PCs are stripped off the preexisting retinal vessels in DR. Importantly, APB5 administration to adult mice failed to induce PC loss from mature retinal vessels (data not shown), indicating that the PDGFR $\beta$ signal is dispensable for the maintenance of EC-PC associations. Moreover, the vascular abnormalities originating from hyperglycemia in DR may not be entirely the same as those induced merely by PC loss. Nevertheless, while the disease progression state of DR can be improved by resolution of hyperglycemia at the initial stages, DR autonomously progresses regardless of the blood glucose levels during the advanced stages. This suggests that intrinsic retinal events cause the deterioration of DR independently of glucose levels, once the self-repairing ability of the retinal vessels becomes disrupted. Notably, we occasionally found differential phenotypes in the right and left eyes, and autonomous disease progression after single APB5 injections, suggesting that the disease irreversibly progressed when stochastic events triggered by PC depletions exceeded a threshold level, which may mimic the susceptibility to retinopathy in individual eyes of diabetic patients. 
A

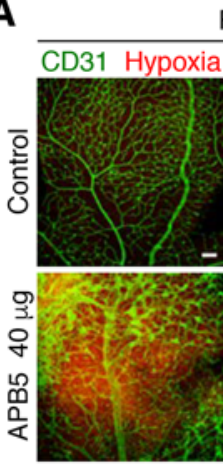

C

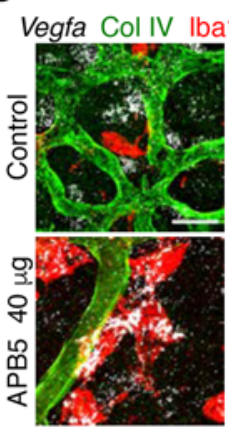

E

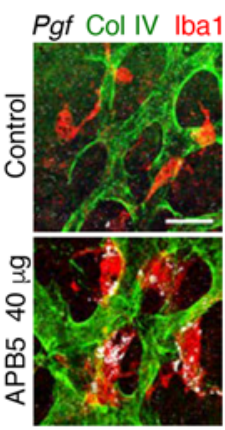

8

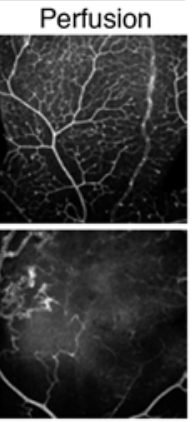

D
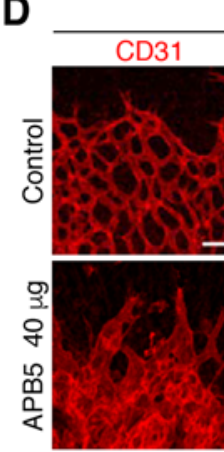

$\mathbf{F}$

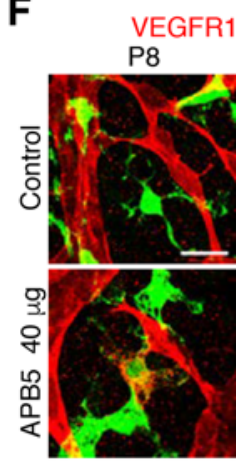

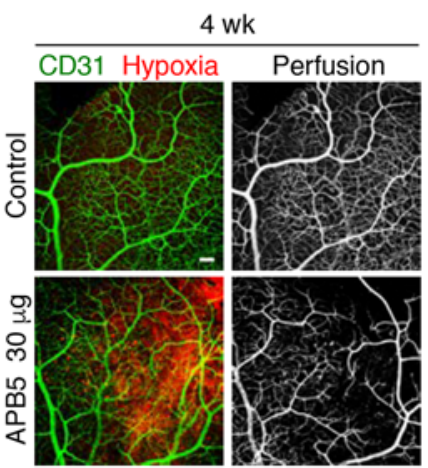

P8

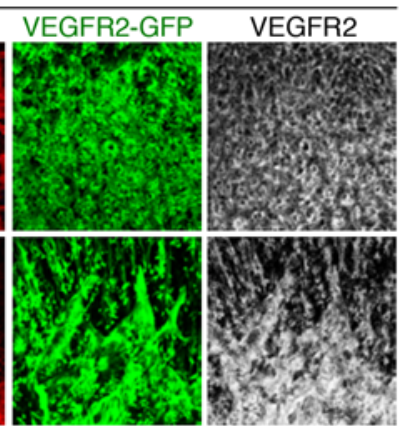

G

B
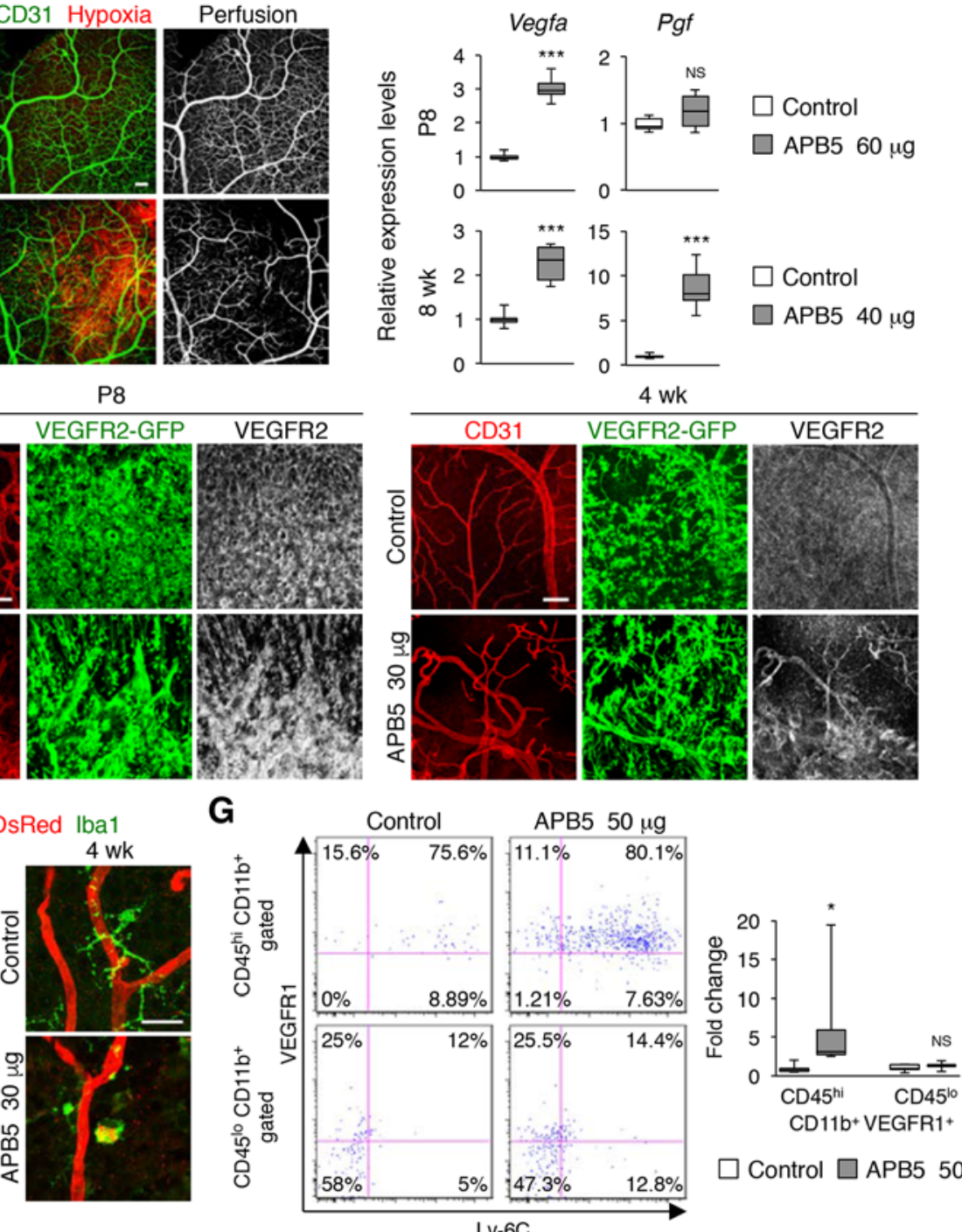

$\square$ Control $\square$ APB5 $50 \mu \mathrm{g}$

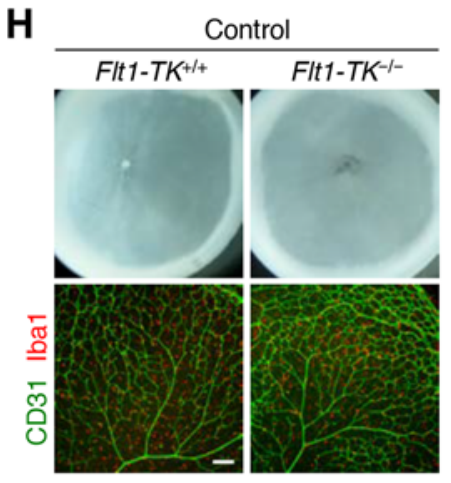

$\square$ Flt1-TK $K^{+/+} \square$ Flt1-TK

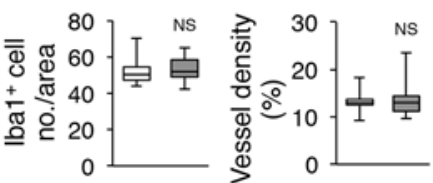
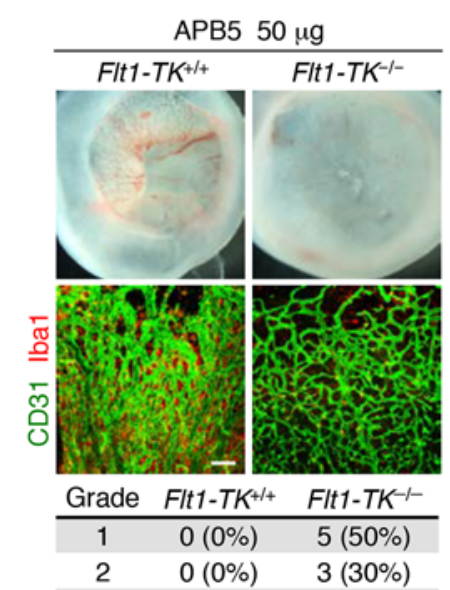

$\begin{array}{lll}3 & 2(20 \%) & 2(20 \%)\end{array}$ \begin{tabular}{rrr}
4 & $8(80 \%)$ & $0(0 \%)$ \\
\hline
\end{tabular}

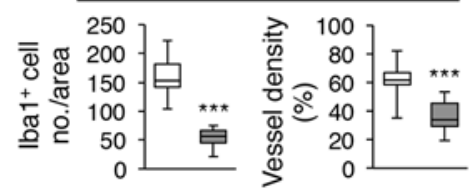

I

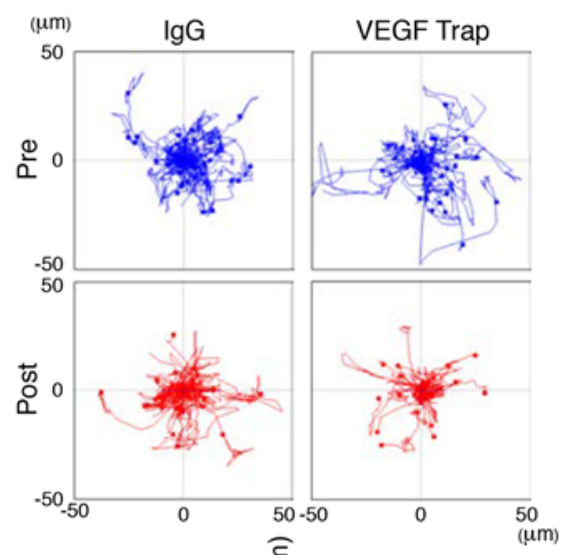

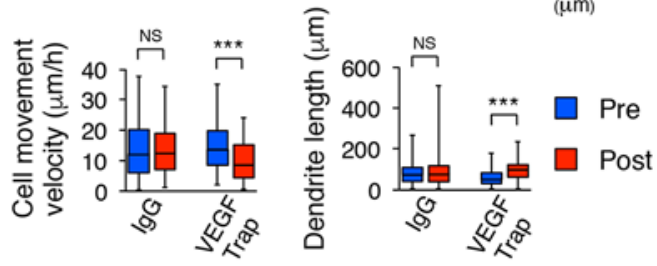


Figure 6. Endothelial cell-macrophage interactions via VEGF and placental growth factor (PIGF). (A) Detection of retinal hypoxia and vessel perfusion at P8 and 4 weeks by i.p. pimonidazole and transcardiac Tomato lectin injections, respectively, along with IHC for CD31. Note the hypoxia in the areas of hypoperfused vessels in the APB-treated retinas. (B) Quantitative reverse transcription PCR (qRT-PCR) of P8 $(n=8)$ and 8-week $(n=6)$ retinas. (C) ISH for Vegfa (white) and IHC for type IV collagen (Col IV) and Iba1 in P8 retinas. (D) IHC for CD31 and VEGFR2 (white) in retinas from P8 and 4-week Vegfr2-BAC-GFP mice. Note the endothelial VEGFR2 upregulation in APB5-treated retinas, in contrast to the constitutive VEGFR2 expression in neurons and Müller glia. (E) ISH for Pgf (white) and IHC for Col IV and Iba1 in P8 retinas. (F) IHC for Iba1 in retinas from P8 and 4-wk Vegfr1-BACDsRed mice. (C) Flow cytometry in P8 retinas. CD45 hiCD11 b+ and CD45 $5^{\circ} \mathrm{CD} 11 \mathrm{~b}^{+}$cells were gated as shown in Figure $5 \mathrm{~A}$. The graph shows fold changes of the number of CD45 hiCD11b+VEGFR $1^{+}$and $C D 45^{10} \mathrm{CD} 11 \mathrm{~b}^{+}$VEGFR1 $1^{+}$cells per retina $(n=5)$. (H) Representative retina cups (upper) and IHC for CD31 and Iba1 (lower) at P11 in FIt1-TK mice. The APB5-treated retinas were graded according to Supplemental Figure 1C. The graphs show the number of Iba1 cells and the vessel density per area (control, $n=12$; APB5, $n=20$ ). (I) The trajectory of GFP+ cells in ex vivo retina imaging from P8 Cx3cr1-GFP mice injected with $50 \mu \mathrm{g}$ of APB5. After 3-hour imaging, retinas were treated with control IgG or VEGF Trap, and further monitored for 3 hours. The graphs show quantification of cell body movement velocity (Pre IgG, $n=68$; Post IgG, $n=56$; Pre VEGF Trap, $n=52$; Post VEGF Trap, $n=47$ ) and total dendrite length per GFP+ ${ }^{+}$cell (Pre IgG, $n=40$; Post IgG, $n=34$; Pre VEGF Trap, $n=43$; Post VEGF Trap, $n=33$ ). See also Supplemental Video 4. ${ }^{*} P<0.05$, ${ }^{* * *} P<0.001$ (2-tailed Student's $t$ test). Scale bars: $100 \mu \mathrm{m}$ (A and $\left.\mathbf{H}\right) ; 50 \mu \mathrm{m}$ (D); $20 \mu \mathrm{m}$ (C, E, and F).

Although the significance of inflammation in DR has been recognized (48), little is known about how inflammation is induced, what types of leukocytes infiltrate, and how they affect vessel integrity in the absence of PCs. In the present study, we showed that PC depletion activated the NFAT pathway in ECs, which is known to upregulate the expression of inflammatory genes (32). Consistently, our transcriptome analyses showed upregulation of its target genes in PC-free ECs, which might facilitate the influx of circulating leukocytes into retinas (Figure 9). The majority of these infiltrating leukocytes were $\mathrm{CD} 45^{\mathrm{hi}} \mathrm{CD} 11 \mathrm{~b}{ }^{+} \mathrm{Ly}-6 \mathrm{C}^{+}$MPs, which were intimately associated with denuded ECs. Notably, NFAT blockade with cyclosporine, and MP elimination with clodronate liposomes suppressed retinal edema after APB5 injection, indicating their contributions to damaging rather than protecting the BRB integrity in PC-free vessels. To our surprise, injections of a small amount of APB5 sustained retinal inflammation even without edema and hemorrhage, suggesting that inflammation may be occultly present after PC loss in asymptomatic retinas of diabetic patients.

In these settings, VEGF and VEGFR2 were continuously upregulated in MPs and ECs, respectively, suggesting an important role of this signaling axis in hyperpermeability and angiogenesis in PC-free retinal vessels. Furthermore, based on the phenotypes of Flt1-TK $K^{-1-}$ mice and the effects of VEGF Trap on MP dynamics, it is likely that MP-derived VEGF and/or P1GF activated VEGFR1 in MPs in an autocrine or paracrine manner, thereby promoting MP migration in the absence of PCs (Figure 9). However, the specific roles of VEGF and PIGF in the VEGFR1 signal in MPs have yet to be fully determined. To address this issue, MP-specific disruptions of these genes in conjunction with APB5 injections should provide useful information that may be relevant to clinical modalities for treating DR.

In addition to the EC-MP and MP-MP interactions via VEGF and PIGF, our results showed ECEC communications via Angpt2 in PC-free retinas. The overall increase of Tie2 phosphorylation in APB5-treated retinas suggested a possibility that the agonistic potential of EC-derived Angpt2 might compensate for the reductions of PC-derived Angpt1. However, endothelial Angpt2 upregulation coincided with FOXO1 nuclear translocation locally in the aneurysm-like structures. Together with the faint expression of Tie1 proteins in PC-free ECs, these results suggested that MP-derived TNF- $\alpha$ and VEGF facilitated Tie1 cleavage, thereby converting Angpt2 into a Tie2 antagonist in localized retinal areas, where FOXO1 activation and Angpt2 upregulation formed a positive feedback loop (Figure 9). The resulting sensitization of ECs to TNF- $\alpha$ and VEGF might exacerbate inflammation and vascular dysfunctions, leading to sustained hyperpermeability, hypoperfusion, and neoangiogenesis. The antiAngpt2 mAb combined with VEGF Trap could stop this damaging cycle, thus restoring the BRB integrity. These results confirmed the possible clinical benefits of drugs targeting Angpt2 or activating Tie2 for preventing the disease progression in DR.

Incorporating our potentially unique methodologies such as FACS-array transcriptome analyses and imaging in mouse retinas, our experimental model provides new opportunities for drug discovery, not only for $\mathrm{DR}$, but also for neurological disorders in which PC deficiency, inflammation, and blood-brain barrier breakdown are implicated (3). Moreover, given the similarities between retinal pericytes and glomerular mesangial cells in terms of the dependence of their development on the PDGFB/PDGFR $\beta$ signal, and their abnormalities in diabetic patients (49), our retinal research may further contribute to understanding the common mechanisms underlying diabetic microvascular complications. 
A

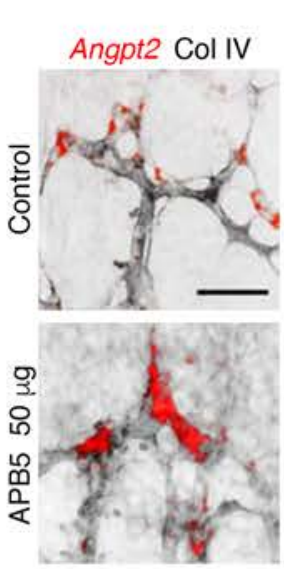

D
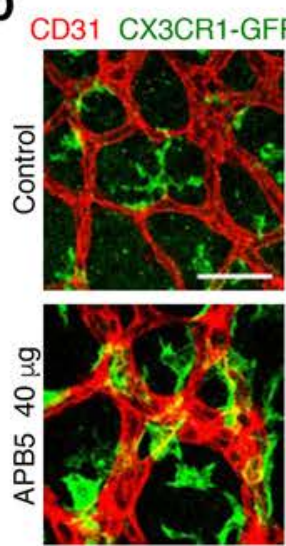

$\mathbf{F}$

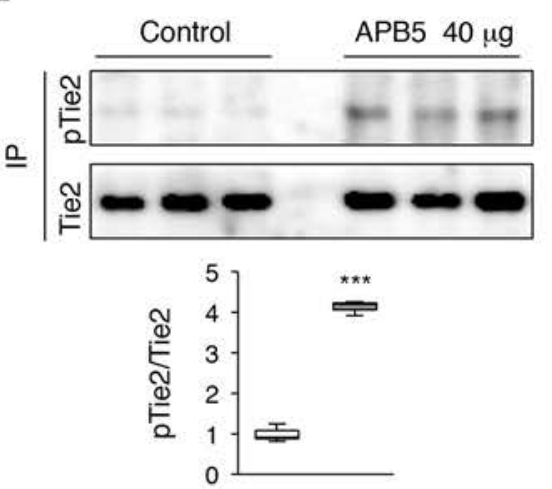

$\square$ Control $\square$ APB5 $40 \mu \mathrm{g}$
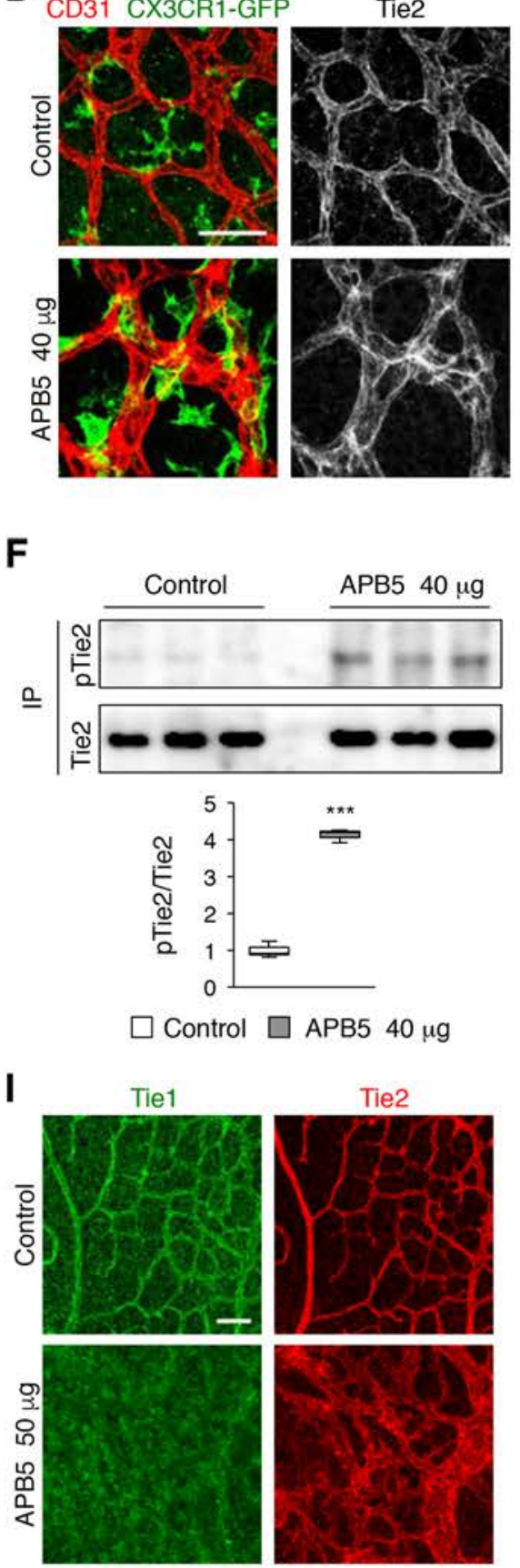
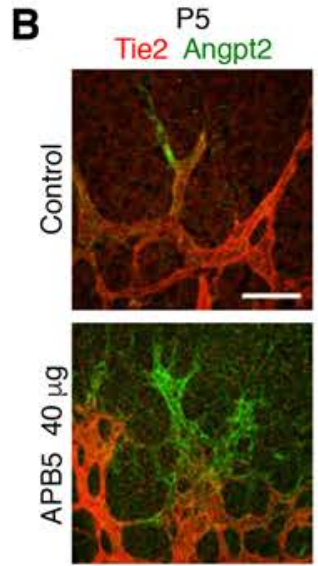

E

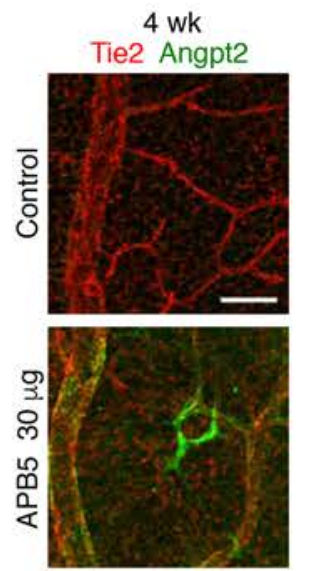

C

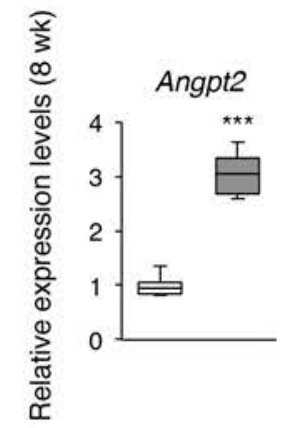

$\square$ Control $\square$ APB5 $40 \mu \mathrm{g}$
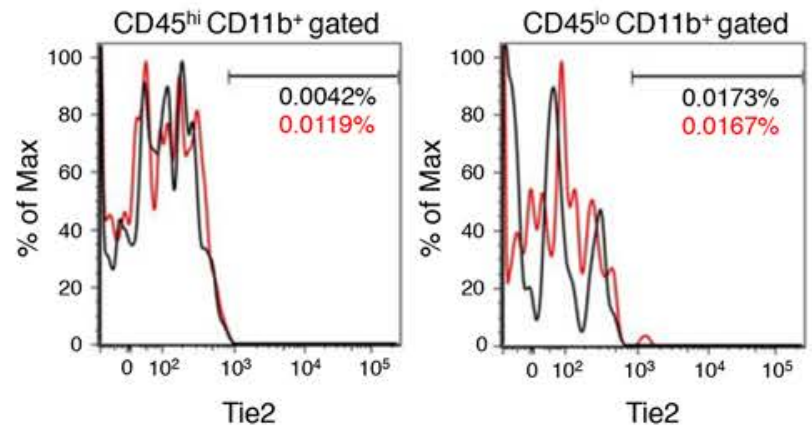

- Control

- APB5 $50 \mu \mathrm{g}$

G
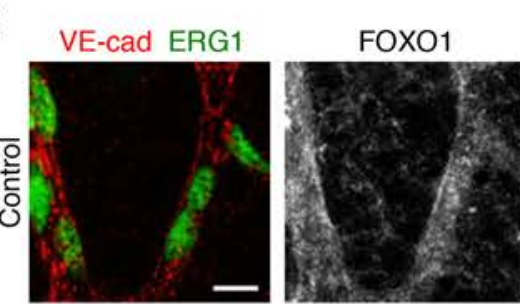

H FOX01 Angpt2
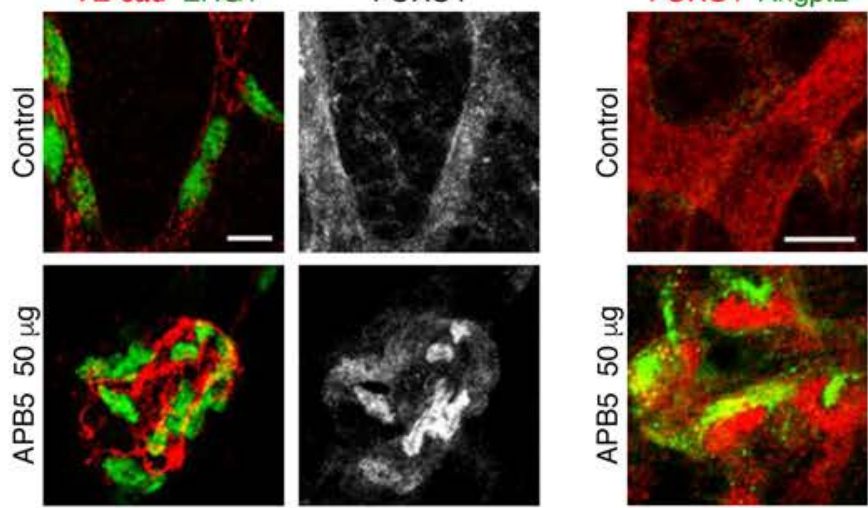

J

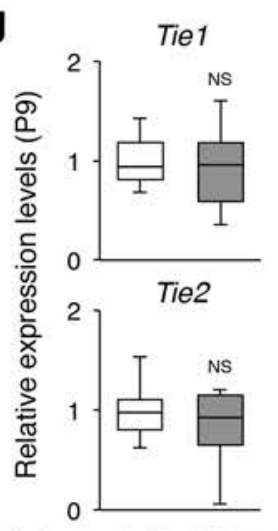

K
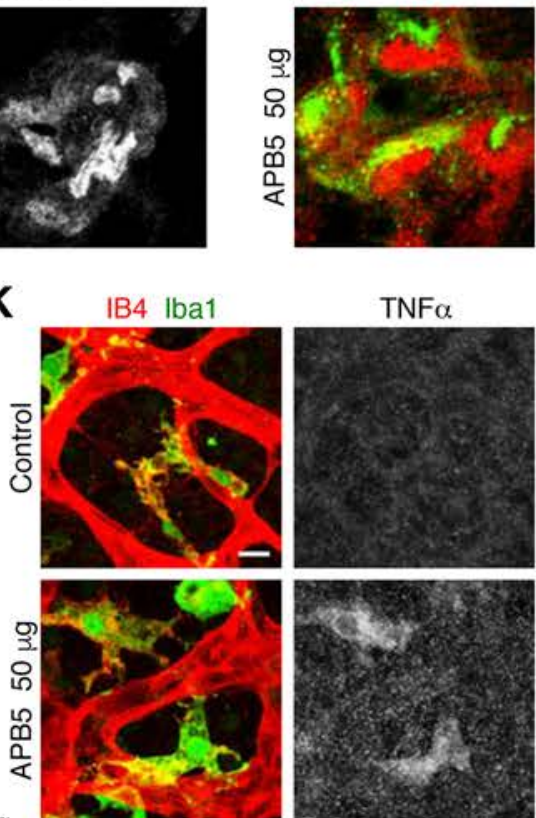

$\square$ Control $\square$ APB5 $60 \mu \mathrm{g}$ 
Figure 7. Angiopoietin-2 (Angpt2) upregulation and FOX01 activation in pericyte-free endothelial cells. (A) ISH for Angpt2 and IHC for type IV collagen (Col IV) in P4 retinas. Note the Angpt2 upregulation in stalk endothelial cells (ECs) of APB5-treated retinas. (B) IHC for Tie2 and Angpt2 in P5 and 4-week retinas. Note their complementary expression patterns in pericyte-free ECs. (C) Quantitative reverse transcription PCR (qRT-PCR) of 8-week retinas ( $n=6$ ). (D) IHC for CD31 and Tie2 in retinas of P8 Cx3cr1-GFP mice. Tie2 expression was absent in GFP+ cells both in the control and APB5-treated retinas. (E) Flow cytometry

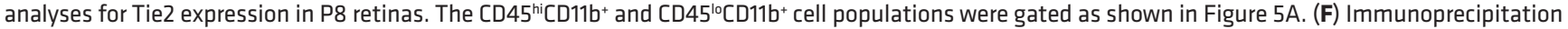
(IP) and immunoblotting of phosphorylated Tie2 (pTie2) and Tie2 in P8 retinas. The graph shows quantification of pTie2/Tie2 ( $n=3$ retinas). (G and $\mathbf{H})$ IHC for vascular endothelial cadherin (VE-cad), Ets-related gene-1 (ERG1), and FOXO1 (G), and Angpt2 and FOX01 (H) in P9 retinas. Note the FOXO1 nuclear translocation in Angpt2-expressing ECs of aneurysm-like structures in the APB5-treated retinas. (I) IHC for Tie1 and Tie2 in P9 retinas. (J) The qRT-PCR of P9 retinas $(n=6)$. (K) IHC for IB4, Iba1, and TNF- $\alpha$ in P9 retinas. ${ }^{* *} P<0.001$ (2-tailed Student's $t$ test). Scale bars: $50 \mu \mathrm{m}(\mathbf{A}, \mathbf{B}, \mathbf{D}$, and I); $10 \mu \mathrm{m}(\mathbf{G}, \mathbf{H}$, and $\mathbf{K})$.

\section{Methods}

Animals. Unless otherwise specified, all experiments were performed using C57BL/6 WT mice (Japan SLC). For the Evans Blue and acridine orange experiments, ICR WT mice (Japan SLC) were used. For reporter strains, the homozygous Tie2-GFP mice (29) (The Jackson Laboratory) and the heterozygous Cx3cr1-GFP (36) (The Jackson Laboratory), Dscr-1-lacZ-Hprt (31), Vegfr2-BAC-GFP (39), and Vegfr1-BAC-DsRed (40) mice were used. The homozygous $F l t 1-T K^{-/-}$mice (41) were obtained by mating heterozygous breeders.

Injections of reagents. A rat anti-mouse PDGFR $\beta$ mAb (clone APB5) (7) dissolved in PBS at $1 \mathrm{mg} / \mathrm{ml}$ was i.p. injected once at P1, except for daily i.p. injections in FACS-array analyses. The amount of APB5 was optimized to control the extents of PC depletion and subsequent vascular leakage and inflammation in retinas, depending on the purpose of each experiment. For controls, the same volume of PBS was injected into the littermates. To block the NFAT signal and deplete MPs, $50 \mu 1$ of $10 \mathrm{mg} / \mathrm{ml}$ cyclosporine (Merck Millipore) dissolved in 1:9 ethanol/sunflower oil (Sigma-Aldrich), and $100 \mu 1$ of $7 \mathrm{mg} / \mathrm{ml}$ clodronate liposomes (FormuMax) were i.p. injected, respectively. Their littermates were injected with the same volume of control vehicle or liposomes. For intravitreal injections, $1 \mu \mathrm{l}$ of $2.5 \mathrm{mg} / \mathrm{ml}$ human polyclonal IgG, humanized anti-Angpt2 mAb (45), VEGF Trap (42), or a mixture of anti-Angpt2 mAb and VEGF Trap (2.5 mg/ $\mathrm{ml}$ for each) was injected using a glass capillary pipette equipped with a micromanipulator (Drummond Scientific). Anti-Angpt $2 \mathrm{mAb}$ and VEGF Trap were provided from Gou Young Koh (Korea Advanced Institute of Science and Technology, Daejeon, Republic of Korea).

Histological analyses. IHC of whole-mount and cryosectioned retinas was processed after fixation with $4 \%$ paraformaldehyde (PFA) in PBS, as previously described (7). The primary Abs and reagents are listed in Supplemental Table 2. The secondary Abs were donkey and goat IgG conjugated with Alexa Fluor 488 (Thermo Fisher Scientific), Cy3, Cy5, or DyLight 649 (Jackson ImmunoResearch). Nuclei were stained with TO-PRO-3 (Thermo Fisher Scientific). H\&E staining in paraffin-embedded retinal sections was performed as previously described (7). For $\beta$-gal staining, retinas were fixed in $0.1 \mathrm{M}$ phosphate buffer (PB; $\mathrm{pH}$ 7.2) containing $0.2 \%$ glutaraldehyde, $5 \mathrm{mM}$ EGTA, and $0.5 \mathrm{mM} \mathrm{MgCl}$, and rinsed in $0.1 \mathrm{M} \mathrm{PB}$ containing $0.02 \% \mathrm{NP}-40$ and $0.5 \mathrm{mM} \mathrm{MgCl}_{2}$. Retinas were then incubated in $0.1 \mathrm{M} \mathrm{PB}$ containing $1 \mathrm{mg} /$ $\mathrm{ml}$ Bluo-Gal (Thermo Fisher Scientific), $50 \mathrm{mM}$ potassium ferrocyanide, $50 \mathrm{mM}$ potassium ferricyanide, and $0.5 \mathrm{mM} \mathrm{MgCl}_{2}$. For proliferation assays, $30 \mu \mathrm{g} / \mathrm{g}$ body weight (BW) of 5-ethynyl-2'-deoxyuridine (EdU) was i.p. injected 2 hours prior to sacrifice. The retinas were processed with a Click-iT EdU Alexa Fluor 488 imaging kit (Thermo Fisher Scientific) according to the manufacturer's instructions. Wholemount ISH using a digoxigenin-labeled cRNA probe for Angpt2, and RNAscope probes (Advanced Cell Diagnostics) for $V e g f a$ and $P g f$ was performed as previously reported $(43,50)$. Images were taken using an LSM700 confocal microscope (Zeiss) with ZEN software (Zeiss), an Axioplan 2 microscope (Zeiss) with AxioVision software (Zeiss), or a BZ-X710 microscope (Keyence) with BZ-X software (Keyence). Retina cups were observed with an M205C stereoscopic microscope (Leica) equipped with a DP21 digital camera (Olympus). Images were analyzed using NIH ImageJ (version 1.49), Adobe Photoshop (Adobe Systems), or IMARIS (Bitplane) software.

Morphological analyses. All analyses were performed in whole-mount retinas. The vessel density and PC coverage were calculated as the proportions of $\mathrm{CD} 31^{+}$and $\mathrm{NG} 2^{+}$areas, respectively, in four $320 \times$ $320 \mu \mathrm{m}$ (neonate) or two $500 \times 500 \mu \mathrm{m}$ (adult) fields per retina. The radial vascular extension per retina was calculated by averaging the distances from the optic nerve to the angiogenic front in 4 retinal quadrants. The artery and vein diameters were calculated by averaging the values at 4 to 5 points per vessel, at approximately $200-300 \mu \mathrm{m}$ from the optic nerve in neonates, and in the central retinal areas in adults. The capillary diameter was measured in 20 (neonate) or 10 (adult) capillary branches per retina. The filopodia lengths and filopodia numbers per $100 \mu \mathrm{m}$ vessel length were measured in sprouting vascular 
A

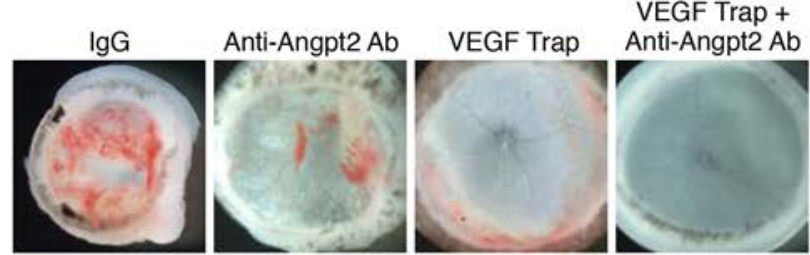

\begin{tabular}{ccccc} 
Grade & IgG & Anti- Angpt2 Ab & VEGF Trap & $\begin{array}{c}\text { VEGF Trap + } \\
\text { Anti-Angpt2 Ab }\end{array}$ \\
\hline 1 & $0(0 \%)$ & $2(6 \%)$ & $9(30 \%)$ & $16(55 \%)$ \\
2 & $1(3 \%)$ & $1(3 \%)$ & $14(47 \%)$ & $9(31 \%)$ \\
3 & $4(13 \%)$ & $12(39 \%)$ & $4(13 \%)$ & $2(7 \%)$ \\
4 & $26(84 \%)$ & $16(52 \%)$ & $3(10 \%)$ & $2(7 \%)$ \\
\hline
\end{tabular}

B
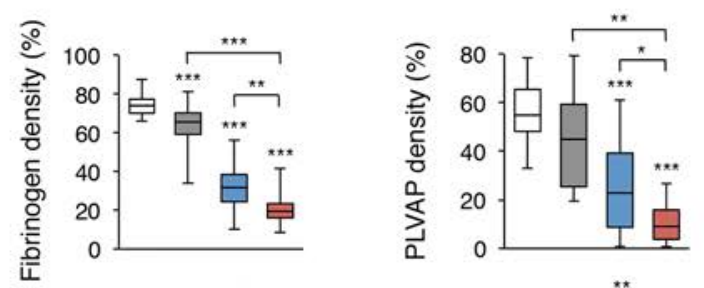

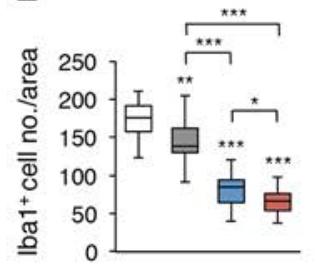

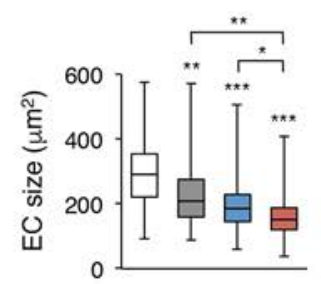

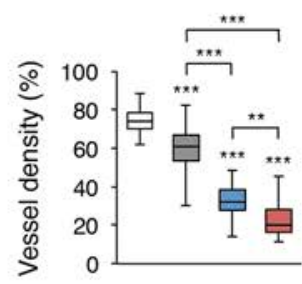

$\square \lg G$

$\square$ Anti-Angpt2 Ab

$\square$ VEGF Trap

$\square$ VEGF Trap + Anti-Angpt2 Ab
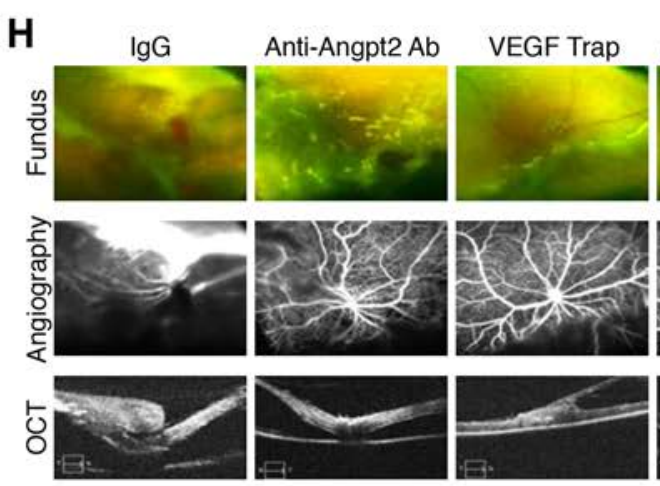

VEGF Trap + Anti-Angpt2 $\mathrm{Ab}$

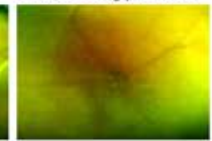

C

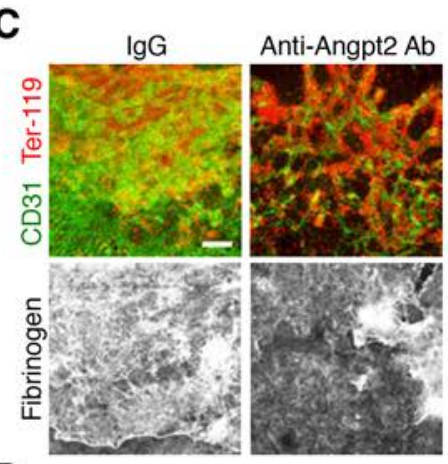

D
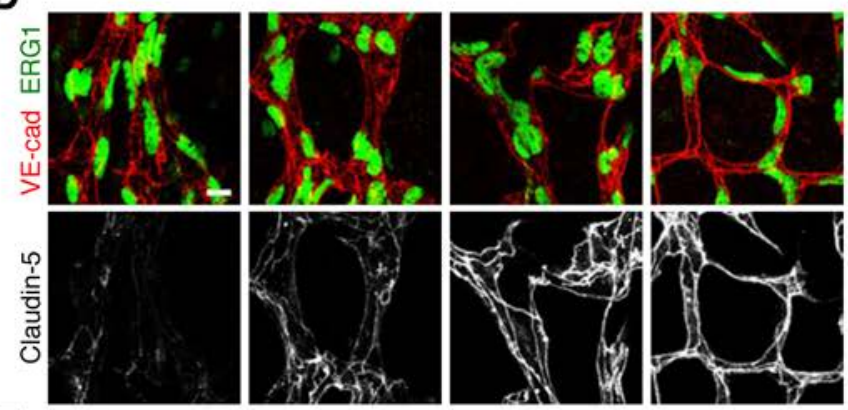

E
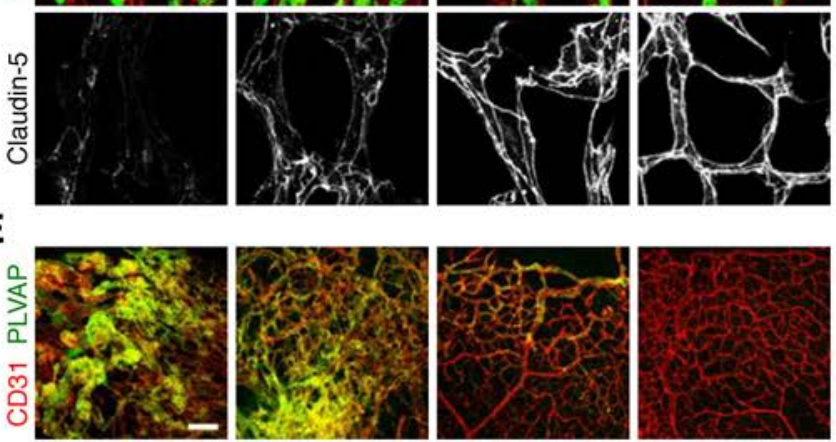

$\mathbf{F}$
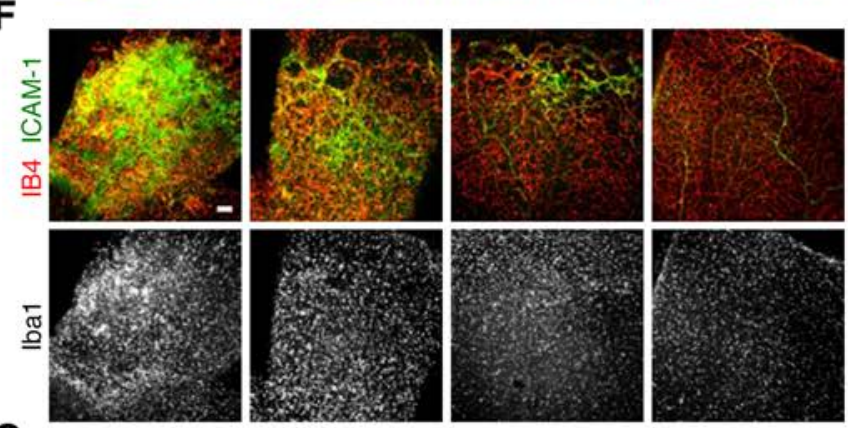

G
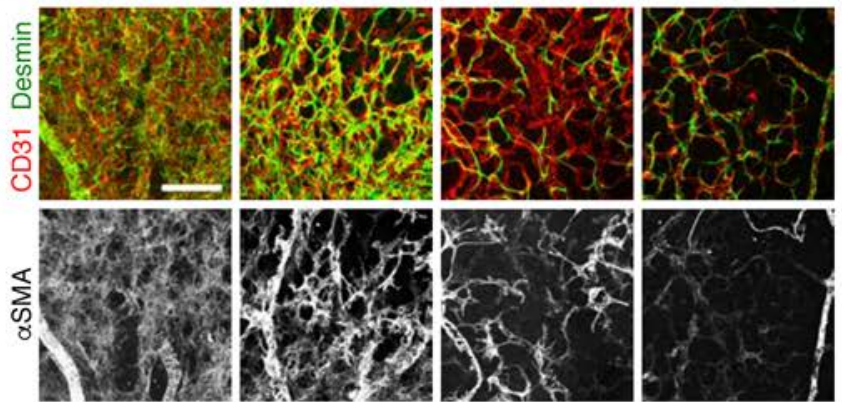

Figure 8. Restoration of retinal vessel integrity by neutralizing VEGF, placental growth factor (PIGF), and angiopoietin-2 (Angpt2). Retinas were analyzed after i.p. APB5 injections (50 $\mu \mathrm{g}$ ) at P1, and intravitreal drug injections at P7. (A) Representative retina cups at P13 and their grading. (B) Quantification of fibrinogen density $\left(n=20\right.$ areas), plasmalemma vesicle-associated protein (PLVAP) density ( $n=16$ areas), Iba $1^{+}$cell number $(n=20$ areas), endothelial cell (EC) size $(n=160)$, and vessel density $(n=20$ areas) in P13 retinas. (C-C) IHC for CD31, Ter-119, and fibrinogen (C), Ets-related gene-1 (ERG1), vascular endothelial cadherin (VE-cad), and claudin-5 (D), CD31 and PLVAP (E), IB4, ICAM-1, and Iba1 (F), and CD31, desmin, and $\alpha$-smooth muscle actin ( $($ SMA) (C) in P13 retinas. (H) Retina imaging for color fundus photography, fluorescence angiography, and optical coherence tomography (OCT) in 4-week retinas. ${ }^{*} P<0.05,{ }^{* *} P<0.01,{ }^{* *} P<0.001$ (1-way ANOVA). Scale bars: $100 \mu \mathrm{m}$ (C and E-C); $10 \mu \mathrm{m}$ (D). 


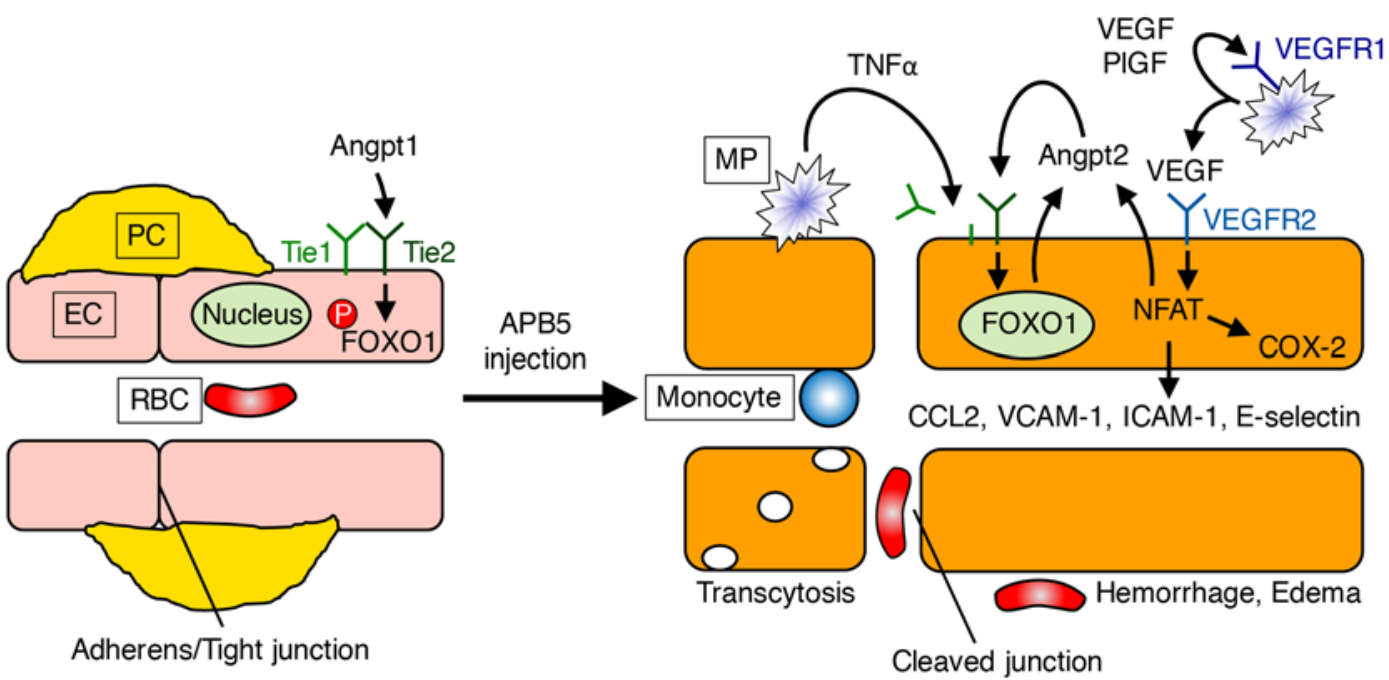

Figure 9. Schematic diagram of irreversible blood-retina barrier (BRB) breakdown in pericyte-free retinal vessels. In the presence of pericytes (PCs), angiopoietin-1 (Angpt1)-induced Tie2 activation phosphorylates FOXO1 and inhibits its nuclear translocation in endothelial cells (ECs). After PC depletion, endothelial NFAT activation upregulates inflammatory chemokines, leukocyte adhesion molecules, and Angpt2, which together promote the influx of circulating monocytes into retinas. The infiltrating macrophages (MPs) secrete VEGF and placental growth factor (PICF), which activate VEGFR1 in MPs and VEGFR2 in ECs. The VEGF/VEGFR2 signal further activates NFAT and upregulates Angpt2. MP-derived VEGF and TNF- $\alpha$ facilitate Tie1 cleavage and Angpt2 conversion from a Tie2 agonist into an antagonist, which then activates FOXO1 and upregulates its target genes including Angpt2. This positive feedback loop renders ECs more sensitive to VEGF and TNF- $\alpha$, and sustains inflammation and vascular dysfunction. The resulting hypoxia further upregulates VEGF and Angpt2, leading to the irreversible BRB breakdown with paracellular and transcellular leakage and RBC extravasation.

fronts. The total number of aneurysms and artery-vein crossings were counted per retina. The morphometry of individual ECs was analyzed in retinas labeled for VE-cadherin and Ets-related gene-1. The number of ECs per area was counted in four $160 \times 160 \mu \mathrm{m}$ fields per retina. The numbers of EdU ${ }^{+}$ and cleaved caspase- $3^{+}$ECs per area were counted in four $320 \times 320 \mu \mathrm{m}$ fields per retina in neonates. In adults, the total numbers of ECs with EdU uptake, filopodia projections, and caspase- 3 expression were counted per retina. The number of $\mathrm{CD}^{2} 1^{-}$collagen $\mathrm{IV}^{+}$vessel sleeves per area was counted in four 500 $\times 500 \mu \mathrm{m}$ fields per retina. The number of MPs per area was counted in four (neonate) or two (adult) $500 \times 500 \mu \mathrm{m}$ fields per retina. The fibrinogen, PLVAP, and vessel densities after drug injections were calculated in four $500 \times 500 \mu \mathrm{m}$ fields per retina. The EC size after drug injections was measured in 40 capillary ECs per retina.

Assays for vascular permeability, perfusion, and hypoxia. For the permeability assay, $100 \mu 1$ of a $2 \%$ Evans Blue dye (Sigma-Aldrich) was i.p. injected 24 hours prior to perfusion fixation with 4\% PFA in PBS. To visualize tracer permeability, $30 \mu \mathrm{l}$ (P8) or $100 \mu \mathrm{l}$ (4 weeks) of $1 \mathrm{mg} / \mathrm{ml}$ EZ-Link Sulfo-NHS-Biotin (Thermo Fisher Scientific) was injected into the heart 10 minutes prior to perfusion fixation with $1 \%$ PFA in PBS. The extravasated biotin was detected with Cy3-conjugated anti-biotin $\mathrm{mAb}$ (Sigma-Aldrich). To detect vessel perfusion and hypoxia, $60 \mu \mathrm{g} / \mathrm{g}$ BW of pimonidazole hydrochloride (Hypoxyprobe) was i.p. injected 60 minutes prior to transcardiac perfusion of DyLight 594-conjugated Lycopersicon esculentum (Tomato) lectin (Vector Laboratories). After 10 minutes, retinal samples were obtained following perfusion fixation with 4\% PFA in PBS, and stained with anti-pimonidazole Ab (Hypoxyprobe).

Electron microscopy. For transmission electron microscopy, mice were perfused with $2 \%$ PFA and $2 \%$ glutaraldehyde in $0.1 \mathrm{M} \mathrm{PB}(\mathrm{pH}$ 7.4), and whole-mount retinas were further incubated in the same fixative overnight at $4^{\circ} \mathrm{C}$. For visualizing the tracer permeability, either $30 \mu \mathrm{l}(\mathrm{P} 8)$ or $100 \mu \mathrm{l}$ (4 weeks) of $1 \mathrm{mg} / \mathrm{ml}$ EZ-Link Sulfo-NHS-biotin was i.v. injected 60 minutes prior to perfusion fixation. Whole-mount retinas were fixed, frozen in OCT compound, and cryosectioned to a thickness of $14 \mu \mathrm{m}$. The specimens were sequentially incubated with $3 \% \mathrm{H}_{2} \mathrm{O}_{2}$, a VECTASTAIN Elite ABC standard kit (Vector Laboratories), and an electrondense DAB kit (Nichirei). Both the whole-mount and sectioned samples were postfixed sequentially with $0.1 \mathrm{M} \mathrm{PB}$ ( $\mathrm{pH} 7.4$ ) containing $2 \% \mathrm{PFA}$ and $2 \%$ glutaraldehyde, and with $2 \% \mathrm{OsO}_{4}$, dehydrated in a graded ethanol series, placed in propylene oxide, and embedded in Quetol 812 epoxy resin. Sections were cut using 
an Ultracut-S microtome (Leica) with a diamond knife, stained with modified Sato's lead solution (51) for 30 seconds, and examined under a JEM-1011J electron microscope (JEOL). Scanning electron microscopy was performed as previously reported (52), with slight modifications. Briefly, retinas were fixed in $2.5 \%$ glutaraldehyde and 2\% PFA in $0.1 \mathrm{M} \mathrm{PB}(\mathrm{pH} 7.4)$, treated with $1.0 \% \mathrm{NaClO}$ for 20 seconds at room temperature, and hydrolyzed with $8 \mathrm{~N} \mathrm{HCl}$ for 30 minutes at $60^{\circ} \mathrm{C}$. After a brief rinse, the specimens were dehydrated using a graded ethanol series, immersed in isoamyl acetate, freeze-dried using a tert-butyl alcohol dryer (Vacuum Device), coated with platinum, and observed with an S-800 scanning electron microscope (Hitachi).

Electroretinogram (ERG). After overnight dark adaptation, mice were deeply anesthetized followed by pupil dilation with a Mydrin-P eye drop (Santen Pharmaceutical). Using a monopolar contact lens electrode (Mayo Corporation) placed on the cornea, full-field ERGs were elicited by white light flashes at intensities from $4.592 \times 10^{-5}$ to $2.559 \mathrm{~cd} \mathrm{~s} / \mathrm{m}^{2}$ with a duration of $25 \mathrm{~ms}$ using a Ganzfeld Stimulator (LKC Technologies) with attenuated neutral density filters. The ERGs were differentially amplified and filtered from 0.3 to $500 \mathrm{~Hz}$ by a Bio Amplifier ML132 (AD Instruments). The amplified responses were acquired at a $4 \mathrm{kHz}$ sampling rate by the Power Lab 2/25 (AD Instruments). The amplitude of the a-wave was measured from the baseline to the a-wave trough. The amplitude of the $b$-wave was measured from the a-wave trough to the $b$-wave peak. Because the acquired ERGs of the $a$ - and b-waves were superimposed on oscillatory potentials, the original signal was processed using the Fast Fourier Transform method with a $60 \mathrm{~Hz}$ cutoff frequency to extract only the a- and b-waves to measure their wave amplitudes.

Widefield fundus photography and optical coherence tomography. After pupil dilation, images of retinal color fundus, fluorescein angiography, and leukocyte labeling were captured using the Optos200TX imaging system (Optos). For angiography, images were taken 2-4 minutes after i.p. injections of fluorescein sodium (Alcon). Retinal leukocytes were detected 2 hours after i.p. injections of $10 \mu \mathrm{l} / \mathrm{g} \mathrm{BW}$ of acridine orange (Wako). The number of leukocytes per area was counted in 2 fields of $752 \times 600$ pixels per retina. The optical coherence tomography images were acquired using the Cirrus HD-OCT (Zeiss) or the OCT2 (Phoenix Research Lab). The retinal and outer nuclear layer thicknesses were measured at approximately $300 \mu \mathrm{m}$ from the optic disk.

Intravital retina imaging. Immediately after i.v. injection of $50 \mu 1$ of $0.2 \%$ Evans Blue dye, intravital retina imaging of the 4-week Cx3cr1-GFP mice was performed under anesthesia and pupil dilation. A contact lens customized for mouse eyes was placed on the cornea with hypromellose (Samil Pharmaceuticals) to prevent acute lens opacity under anesthesia. During the entire imaging experiments, the body temperature was maintained at $37^{\circ} \mathrm{C}$ with a heating pad and temperature sensor (Kent Scientific). Intravital retina imaging used a custom-built multicolor video-rate laser-scanning confocal microscope modified for mouse retinas (53). Two scanning mirrors, a rotating polygonal mirror (Lincoln Laser) and a galvanometer-based scanning mirror (Cambridge Technology), were used to generate a 2-dimensional (2D) laser-scanning pattern, which was delivered to the sample through the relay lenses. The descanned fluorescent light collected by a $20 \times$ objective lens (Nikon) was detected using multi-alkali photocathode photomultiplier tubes (Hamamatsu). The electrical signal was digitized by a frame grabber (Matrox) and reconstructed to images with a size of $512 \times 512$ pixels. The time-lapse images were acquired for 1 hour with a 1-minute interval. Motion artifacts caused by breathing and heart beating were corrected to enhance the signal-to-noise ratio by using a custom-written MATLAB code based on a 2D translational image registration algorithm (54). The skeletal structures of the CX3CR1-GFP ${ }^{+}$cells were automatically analyzed with the Filament Tracer module of IMARIS. The total dendrite length was calculated as the sum of the filament length of each cell. The dendrite motility was calculated as the mean speed of terminal points of each dendrite in the time-series images.

Ex vivo retina imaging. Retinas from P8 Cx3cr1-GFP mice were mounted in collagen gels prepared from an acid collagen solution (Koken) according to the manufacturer's protocol, in conjunction with 1:500 Alexa Fluor 568-conjugated isolectin B4 (Thermo Fisher Scientific). The retinas were incubated in DMEM/F-12 culture medium (Nacalai Tesque) containing 10\% FBS, 500 ng/ml FGF2 (Peprotech), and 1:5,000 isolectin $\mathrm{B} 4$. The culture dish was kept in a chamber $\left(37^{\circ} \mathrm{C}, 5 \% \mathrm{CO}_{2}\right.$, and $\left.40 \% \mathrm{O}_{2}\right)$ on the stage of a CSU-X1 spinning-disk confocal microscope (Yokogawa) equipped with an iXon + CCD camera (Andor). Five-minute interval time-lapse images with a dimension of $512 \times 512$ pixels were taken using a water immersion 20× objective lens (Olympus). After a 3-hour recording, human polyclonal IgG or VEGF Trap $(400 \mu \mathrm{g} / \mathrm{ml})$ was added to the medium, and the recording was continued for 3 hours. Images were analyzed using MetaMorph software (Molecular Devices) and NIH ImageJ software. The dendrite 
length and motility of the CX3CR1-GFP ${ }^{+}$cells were quantified with IMARIS software as describe above. For the analyses of cell body motility, the centroid of each CX3CR1-GFP ${ }^{+}$cell was located in the timeseries images by the Spot Object tool of IMARIS.

Quantitative reverse transcription PCR ( $q R T-P C R)$. From $1 \mu \mathrm{g}$ of total RNA extracted from a single retina using the RNeasy Mini Kit including on-column DNase digestion (Qiagen), the cDNAs were synthesized using Superscript Vilo transcriptase (Thermo Fisher Scientific). The qRT-PCR analyses were performed using the Quantitect SYBR Green PCR Mastermix (Qiagen), followed by quantitative measurements as previously described (55). The TATA box-binding protein was used as an internal control. The primer sequences are shown in Supplemental Table 3.

FACS and microarray analyses. $\mathrm{GFP}^{+} \mathrm{ECs}$ were sorted from retinas of Tie2-GFP mice using a FACSAria cell sorter (BD Biosciences), and the RNA was purified using the RNeasy Micro Kit (Qiagen), followed by linear amplification using the MessageAmpII aRNA amplification kit (Thermo Fisher Scientific), as previously reported (30). Biotin-labeled cRNA preparations and hybridization onto the Affymetrix oligonucleotide arrays (MGU74v2) were performed as previously described (56). The annotation of probe sets was performed using the information provided in the Affymetrix standard annotation file (http://www.affymetrix.com). The CEL files were normalized with the perfect match-only model using dChip software, version 1.3 (57). All data were MIAME compliant and were deposited in the NCBI Gene Expression Omnibus database and are accessible through GEO series accession numbers GSE27238 (http://www.ncbi.nlm.nih.gov/geo/query/acc.cgi?acc=GSE27238) and GSE86819 (https://www.ncbi.nlm.nih.gov/geo/query/acc.cgi?acc=GSE86819). To identify genes differentially expressed between the 2 groups, the significance analysis of microarrays (58) was used with a cutoff threshold of 2-fold changes and a median FDR less than 0.01. For flow cytometry analyses, single-cell suspensions of retinas were incubated with anti-mouse CD16/32 mAb (BioLegend) for 15 minutes, followed by labeling with fluorescence-conjugated mAbs listed in Supplemental Table 2. Staining with 7-aminoactinomycin D (BD Biosciences) was used to eliminate dead cells. Analyses were performed using a FACSCanto II (BD Biosciences) and FlowJo software (Tree Star).

Immunoprecipitation and immunoblotting. Retinas were lysed with RIPA buffer (150 mM NaCl, $50 \mathrm{mM}$ Tris- $\mathrm{HCl}$ [pH 7.5], $10 \mathrm{mM}$ EDTA, and 1\% NP-40) containing Protease Inhibitor Cocktail (Nacalai Tesque), Phosphatase Inhibitor Cocktail (Nacalai Tesque), and $1 \mathrm{mM}$ DTT (Wako). After centrifugation at 13,000 $g$ for 10 minutes at $4^{\circ} \mathrm{C}$, the lysates were incubated overnight at $4^{\circ} \mathrm{C}$ with Protein $\mathrm{G}$ Sepharose beads (GE Healthcare) and goat anti-Tie2 $\mathrm{Ab}$ (R\&D Systems). The immunoprecipitates and the total cell lysates were separated by SDS-PAGE, transferred to Immobilon-P membranes (Merck Millipore), and probed with mouse anti-phosphotyrosine (Enzo) and rabbit anti-Tie2 (Santa Cruz Biotechnology) Abs. HRP-conjugated secondary Abs (Wako) and ImmunoStar LD (Wako) were used for detection. Detailed Ab information is listed in Supplemental Table 2. The band intensities were quantified using NIH ImageJ software.

Statistics. Statistical analyses were performed with SPSS statistics software (IBM) using a 2-tailed Student's $t$ test or 1-way ANOVA with a Games-Howell post-comparison test. In box-and-whisker plots, median (line within the box), upper and lower quartile (bounds of the box), with minimum and maximum values (bars) are shown. $P$ values less than 0.05 were considered statistically significant.

Study approval. All mouse procedures and experiments were approved by the Animal Research Committee of Nagoya City University (permit number H26-M19) and the Animal Care Committee of Korea Advanced Institute of Science and Technology (permit number KA2016-01), and were performed according to the Association for Research in Vision and Ophthalmology Statement for the Use of Animals in Ophthalmic and Vision Research. For the use of human retina images, written informed consent was obtained.

\section{Author contributions}

$\mathrm{SO}$ and $\mathrm{KK}$ performed the experiments and analyzed the data. YHa and TMiy developed the ex vivo imaging system. HT, TIO, and TF performed the electron microscopy experiments. YHw, SA, IP, WI, and PK developed the in vivo imaging system and performed the imaging experiments. SK performed the microarray analyses. YF performed the western blotting experiments. HN and HS contributed to the development of the retinopathy mouse model. JM, ME, MH, TMin, MS, and NT provided reagents and mice. YO contributed to the acridine orange experiments. AU conceived the study, designed and performed the experiments, interpreted data, and wrote the manuscript. 


\section{Acknowledgments}

We thank G.Y. Koh (Korea Advanced Institute of Science and Technology, Republic of Korea) for reagents, M. Shudou (Ehime University, Japan) for electron microscopy observations, H. Kudo (Mayo Corporation, Japan) for ERG experiments, and S.I. Nishikawa (AASJ, Japan) for critical comments on the manuscript. This work was supported by Japan Society for the Promotion of Science KAKENHI grants 16H05155 (A. Uemura), 16K15737 (A. Uemura), and 15H04997 (Y. Ogura); AMED grant 16ek0210070h0001 (A. Uemura); and Japan Science and Technology Agency CREST grant "Spontaneous pattern formation ex vivo" (A. Uemura), with additional funding from the Takeda Science Foundation (A. Uemura), the Naito Foundation (A. Uemura), the Daiko Foundation (A. Uemura), the SENSHIN Medical Research Foundation (A. Uemura), and the Bayer Japan Retina Award (A. Uemura).

Address correspondence to: Akiyoshi Uemura, Department of Retinal Vascular Biology, Nagoya City University Graduate School of Medical Sciences, 1 Kawasumi Mizuho-cho, Mizuho-ku, Nagoya 467-8601, Japan. Phone: 81.52.853.8251; E-mail: uemura@med.nagoya-cu.ac.jp.

1. Armulik A, Genové G, Betsholtz C. Pericytes: developmental, physiological, and pathological perspectives, problems, and promises. Dev Cell. 2011;21(2):193-215.

2. Daneman R, Prat A. The blood-brain barrier. Cold Spring Harb Perspect Biol. 2015;7(1):a020412.

3. Zhao Z, Nelson AR, Betsholtz C, Zlokovic BV. Establishment and Dysfunction of the Blood-Brain Barrier. Cell. 2015;163(5):1064-1078.

4. Cogan DG, Toussaint D, Kuwabara T. Retinal vascular patterns. IV. Diabetic retinopathy. Arch Ophthalmol. 1961;66:366-378.

5. Antonetti DA, Klein R, Gardner TW. Diabetic retinopathy. N Engl J Med. 2012;366(13):1227-1239.

6. Uemura A, Kusuhara S, Katsuta H, Nishikawa S. Angiogenesis in the mouse retina: a model system for experimental manipulation. Exp Cell Res. 2006;312(5):676-683.

7. Uemura A, et al. Recombinant angiopoietin-1 restores higher-order architecture of growing blood vessels in mice in the absence of mural cells. J Clin Invest. 2002;110(11):1619-1628.

8. Diabetic Retinopathy Clinical Research Network, et al. Randomized trial evaluating ranibizumab plus prompt or deferred laser or triamcinolone plus prompt laser for diabetic macular edema. Ophthalmology. 2010;117(6):1064-1077.e35.

9. Koch S, Claesson-Welsh L. Signal transduction by vascular endothelial growth factor receptors. Cold Spring Harb Perspect Med. 2012;2(7):a006502.

10. Dejana E, Tournier-Lasserve E, Weinstein BM. The control of vascular integrity by endothelial cell junctions: molecular basis and pathological implications. Dev Cell. 2009;16(2):209-221.

11. Shibuya M. VEGFR and type-V RTK activation and signaling. Cold Spring Harb Perspect Biol. 2013;5(10):a009092.

12. Aiello LP, et al. Vascular endothelial growth factor in ocular fluid of patients with diabetic retinopathy and other retinal disorders. N Engl J Med. 1994;331(22):1480-1487.

13. Khaliq A, et al. Increased expression of placenta growth factor in proliferative diabetic retinopathy. Lab Invest. 1998;78(1):109-116.

14. McAuley AK, et al. Vitreous biomarkers in diabetic retinopathy: a systematic review and meta-analysis. J Diabetes Complicat. 2014;28(3):419-425.

15. Campochiaro PA. Molecular pathogenesis of retinal and choroidal vascular diseases. Prog Retin Eye Res. 2015;49:67-81.

16. Augustin HG, Koh GY, Thurston G, Alitalo K. Control of vascular morphogenesis and homeostasis through the angiopoietinTie system. Nat Rev Mol Cell Biol. 2009;10(3):165-177.

17. Mandriota SJ, Pepper MS. Regulation of angiopoietin-2 mRNA levels in bovine microvascular endothelial cells by cytokines and hypoxia. Circ Res. 1998;83(8):852-859.

18. Oh H, Takagi H, Suzuma K, Otani A, Matsumura M, Honda Y. Hypoxia and vascular endothelial growth factor selectively upregulate angiopoietin-2 in bovine microvascular endothelial cells. J Biol Chem. 1999;274(22):15732-15739.

19. Daly C, et al. Angiopoietin-1 modulates endothelial cell function and gene expression via the transcription factor FKHR (FOXO1). Genes Dev. 2004;18(9):1060-1071.

20. Kim I, Kim JH, Moon SO, Kwak HJ, Kim NG, Koh GY. Angiopoietin-2 at high concentration can enhance endothelial cell survival through the phosphatidylinositol 3'-kinase/Akt signal transduction pathway. Oncogene. 2000;19(39):4549-4552.

21. Daly C, et al. Angiopoietin-2 functions as an autocrine protective factor in stressed endothelial cells. Proc Natl Acad Sci U S A. 2006;103(42):15491-15496.

22. Kim M, et al. Opposing actions of angiopoietin-2 on Tie2 signaling and FOXO1 activation. J Clin Invest. 2016;126(9):3511-3525.

23. Korhonen EA, et al. Tie1 controls angiopoietin function in vascular remodeling and inflammation. J Clin Invest. 2016;126(9):3495-3510.

24. Yabkowitz R, Meyer S, Black T, Elliott G, Merewether LA, Yamane HK. Inflammatory cytokines and vascular endothelial growth factor stimulate the release of soluble tie receptor from human endothelial cells via metalloprotease activation. Blood. 1999;93(6):1969-1979.

25. Watanabe D, et al. Vitreous levels of angiopoietin 2 and vascular endothelial growth factor in patients with proliferative diabetic retinopathy. Am J Ophthalmol. 2005;139(3):476-481.

26. Gerhardt H, et al. VEGF guides angiogenic sprouting utilizing endothelial tip cell filopodia. J Cell Biol. 2003;161(6):1163-1177.

27. Vestweber D. How leukocytes cross the vascular endothelium. Nat Rev Immunol. 2015;15(11):692-704.

28. Inai $\mathrm{T}$, et al. Inhibition of vascular endothelial growth factor (VEGF) signaling in cancer causes loss of endothelial fenestrations, 
regression of tumor vessels, and appearance of basement membrane ghosts. Am J Pathol. 2004;165(1):35-52.

29. Motoike T, et al. Universal GFP reporter for the study of vascular development. Genesis. 2000;28(2):75-81.

30. Kusuhara S, et al. Arhgef15 promotes retinal angiogenesis by mediating VEGF-induced Cdc42 activation and potentiating RhoJ inactivation in endothelial cells. PLoS One. 2012;7(9):e45858.

31. Minami T, et al. The Down syndrome critical region gene 1 short variant promoters direct vascular bed-specific gene expression during inflammation in mice. J Clin Invest. 2009;119(8):2257-2270.

32. Minami T. Calcineurin-NFAT activation and DSCR-1 auto-inhibitory loop: how is homoeostasis regulated? J Biochem 2014;155(4):217-226.

33. Minami T, et al. The calcineurin-NFAT-angiopoietin-2 signaling axis in lung endothelium is critical for the establishment of lung metastases. Cell Rep. 2013;4(4):709-723.

34. Miyamoto K, et al. Prevention of leukostasis and vascular leakage in streptozotocin-induced diabetic retinopathy via intercellular adhesion molecule-1 inhibition. Proc Natl Acad Sci U S A. 1999;96(19):10836-10841.

35. Sedgwick JD, Schwender S, Imrich H, Dörries R, Butcher GW, ter Meulen V. Isolation and direct characterization of resident microglial cells from the normal and inflamed central nervous system. Proc Natl Acad Sci U S A. 1991;88(16):7438-7442.

36. Jung S, et al. Analysis of fractalkine receptor CX(3)CR1 function by targeted deletion and green fluorescent protein reporter gene insertion. Mol Cell Biol. 2000;20(11):4106-4114.

37. Okabe K, et al. Neurons limit angiogenesis by titrating VEGF in retina. Cell. 2014;159(3):584-596.

38. Nakayama M, et al. Spatial regulation of VEGF receptor endocytosis in angiogenesis. Nat Cell Biol. 2013;15(3):249-260.

39. Ishitobi H, et al. Flk1-GFP BAC Tg mice: an animal model for the study of blood vessel development. Exp Anim. 2010;59(5):615-622.

40. Matsumoto K, et al. Study of normal and pathological blood vessel morphogenesis in Flt1-tdsRed BAC Tg mice. Genesis. 2012;50(7):561-571

41. Hiratsuka S, Minowa O, Kuno J, Noda T, Shibuya M. Flt-1 lacking the tyrosine kinase domain is sufficient for normal development and angiogenesis in mice. Proc Natl Acad Sci U S A. 1998;95(16):9349-9354.

42. Holash J, et al. VEGF-Trap: a VEGF blocker with potent antitumor effects. Proc Natl Acad Sci U S A. 2002;99(17):11393-11398.

43. Felcht M, et al. Angiopoietin-2 differentially regulates angiogenesis through TIE2 and integrin signaling. J Clin Invest. 2012;122(6):1991-2005.

44. Baer C, Squadrito ML, Iruela-Arispe ML, De Palma M. Reciprocal interactions between endothelial cells and macrophages in angiogenic vascular niches. Exp Cell Res. 2013;319(11):1626-1634.

45. Han S, et al. Amelioration of sepsis by TIE2 activation-induced vascular protection. Sci Transl Med. 2016;8(335):335ra55.

46. Weinl C, et al. Endothelial SRF/MRTF ablation causes vascular disease phenotypes in murine retinae. J Clin Invest. 2013;123(5):2193-2206.

47. Franco CA, et al. SRF selectively controls tip cell invasive behavior in angiogenesis. Development. 2013;140(11):2321-2333

48. Adamis AP, Berman AJ. Immunological mechanisms in the pathogenesis of diabetic retinopathy. Semin Immunopathol. 2008;30(2):65-84

49. Betsholtz C, Lindblom P, Bjarnegard M, Enge M, Gerhardt H, Lindahl P. Role of platelet-derived growth factor in mesangium development and vasculopathies: lessons from platelet-derived growth factor and platelet-derived growth factor receptor mutations in mice. Curr Opin Nephrol Hypertens. 2004;13(1):45-52.

50. Yang WJ, Hu J, Uemura A, Tetzlaff F, Augustin HG, Fischer A. Semaphorin-3C signals through Neuropilin-1 and PlexinD1 receptors to inhibit pathological angiogenesis. EMBO Mol Med. 2015;7(10):1267-1284.

51. Hanaichi T, Sato T, Iwamoto T, Malavasi-Yamashiro J, Hoshino M, Mizuno N. A stable lead by modification of Sato's method. J Electron Microsc (Tokyo). 1986;35(3):304-306.

52. Fujiwara T, Uehara Y. The cytoarchitecture of the medial layer in rat thoracic aorta: a scanning electron-microscopic study. Cell Tissue Res. 1992;270(1):165-172.

53. Lee JY, et al. In vivo fluorescence retinal imaging following AAV2-mediated gene delivery in the rat retina. Invest Ophthalmol Vis Sci. 2016;57(7):3390-3396.

54. Guizar-Sicairos M, Thurman ST, Fienup JR. Efficient subpixel image registration algorithms. Opt Lett. 2008;33(2):156-158.

55. Tsujino K, et al. Establishment of TSH $\beta$ real-time monitoring system in mammalian photoperiodism. Genes Cells. 2013;18(7):575-588.

56. Takebe A, Era T, Okada M, Martin Jakt L, Kuroda Y, Nishikawa S. Microarray analysis of PDGFR alpha ${ }^{+}$populations in ES cell differentiation culture identifies genes involved in differentiation of mesoderm and mesenchyme including ARID3b that is essential for development of embryonic mesenchymal cells. Dev Biol. 2006;293(1):25-37.

57. Li C, Wong WH. Model-based analysis of oligonucleotide arrays: expression index computation and outlier detection. Proc Natl Acad Sci U S A. 2001;98(1):31-36.

58. Tusher VG, Tibshirani R, Chu G. Significance analysis of microarrays applied to the ionizing radiation response. Proc Natl Acad Sci U S A. 2001;98(9):5116-5121. 